\title{
CSHP Professional Practice Conference 2012: Poster Abstracts / Conférence sur la pratique professionnelle 2012 de la SCPH : Résumés des affiches
}

Sunday, February 5, 2012 • Dimanche 5 février 2012

Facilitated Poster Session: Discussion of original research, award-winning projects, and pharmacy practice projects

Séance animée de presentations par affiches: Discussions sur des projets de recherché originale, des projets primes et des projets dans le domaine de la pratique pharmaceutique

\section{Antimicrobial and Pediatrics}

1. An Outpatient Parenteral Antimicrobial Therapy Program Experience at the University Health Network

2. Evaluation of a Prospective Audit and Feedback Program in Critical Care: A Controlled Interrupted Time Series Analysis (award-see page 76)

3. Antimicrobial Prescribing Practices for Catheter Urine Cultures

4. Oseltamivir Pharmacokinetics in Morbid Obesity (OPTIMO Trial) (award—see page 77)

5. Description of Propofol Use in a Pediatric Intensive Care Unit

6. Development and Implementation of a Pediatric Emergency Department (ED) Asthma Action Plan and Prescription (AAPP) (award-see page 75)

Clinical

1. Providing Comprehensive Assessment and Pharmaceutical Care at the Geriatric Clinic for Parkinson's

2. Performance of Clinical Prediction Tools Used to Predict Major Bleeding in Patients Receiving Anticoagulation Therapy

3. Development of a Clinical Institute Withdrawal Assessment (CIWA) Scale and Treatment Regimen for the Management of Alcohol Withdrawal

4. Implementation of an Insulin Pen Pilot Project in an In-Patient Hospital Setting

5. Organizational Restructuring of Regional Pharmacy Services to Facilitate Pharmacy Practice Model Change (award-see page 75)

6. Drug Use Evaluation of Intravenous Tranexamic Acid: Adherence to Hospital Guidelines and Adverse Events in an Off-label Use in Orthopedic Patients

\section{Patient}

1. Extending Hospital-Based Medication Incident Reporting to Enhance Medication Safety and Continuous Quality Assurance in Pharmacy Practice (award-see page 76)

2. Implementation and Evaluation of a Medication Reconciliation Tool on Internal Hospital Transfer (award—see page 77)

3. Improving Continuity of Care Post-Hospital Discharge: A Needs Assessment of Community Pharmacists

4. Patient Recall of Interaction with a Pharmacist during Hospital Admission

5. How Well Do Pharmacy Clinicians Perform in a Patient Simulation-Based Admission Medication Reconciliation Validation Program?

6. Who's Looking at the BPMH? Perception and Utilization of the Best Possible Medication History in a Tertiary Care Hospital

\section{Education and Research}

1. The Evaluation of a New Hierarchical Teaching Model for Pharmacy Students in Experiential Education

2. Evaluation of the Effectiveness of Online versus Face to Face Interactions in Interprofessional Education

3. An Online e-Learning Patient Education Tool for Solid Organ Transplant Recipients (award-see page 78)

4. Diabetes Education in Pharmacy Residencies across Canada

5. Evaluating the Role of Pharmacist Teachers in Canadian Family Medicine Residency Programs: A Qualitative Analysis (awardsee page 75$)$
6. A Survey of Institutional Pharmacists' Involvement in and Attitudes towards Research (award—see page 75)

Oncology

1. Interactions between Antiretroviral Agents and Chemotherapy Regimens for the Treatment of Lymphoma: A Quick Reference Guide (award - see page 77)

2. Impact of a Drug Access Facilitator in an Outpatient Chemotherapy Clinic

3. High Dose Methotrexate in Adult Oncology Patients: A Case-Control Study Assessing the Risk Association between Drug Interactions and Methotrexate Toxicity

4. Stability of Azacitidine Solutions in Sterile Water for Injection

5. A Pharmacist-Directed Toxicity Management Program for Patients Receiving Capecitabine: Implementation and Evaluation (awardsee page 76)

\section{Monday, February 6, 2012 • Lundi 6 février 2012}

1. In for a Penny, in for a Pound: Evaluation of Factors Affecting Learner Participation in the Pilot "Adapting Pharmacists' Skills and Approaches to Maximize Patients' Drug Therapy Effectiveness" E-learning Primary Health Care Program

2. Moving Practice Forward with Early Exposure Students

3. Provincial Implementation of Accreditation Canada Required Organizational Practices: The Alberta Experience

4. Drugs Obtained by Quebec University Health Centers through Health Canada's Special Access Programme - Descriptive Analysis by the programme de gestion thérapeutique des medicaments

5. Safety and Efficacy of CHOP or R-CHOP for Treatment of Diffuse Large B-cell Lymphoma with Protease Inhibitor or NonProtease Inhibitor Based Antiretroviral Regimens in HIV-Infected Patients: SCULPT Study

6. Drug Access Navigator Database: Improving the Management of Drug Access Cases through the Development and Implementation of a Computerized Database

7. Residents as Preceptors: Development, Implementation and Evaluation of a Pharmacy Summer Student Clinical Experience

8. Imatinib Plasma Trough Concentrations and the Ability to Assess Patient Adherence

9. Évolution de la thromboprophylaxie chez les usagers médicaux dans un institut universitaire

10. 2010-2011 Perspectives on Drug Shortages in Hospitals in Quebec

11. Multicenter Study on Environmental Contamination by Hazardous Drugs in Quebec Healthcare Centers

12. Temps associé à la purge des tubulures lors de la préparation d'une dose de médicament dangereux et contamination visuelle

13. Is Vasopressin (> 0.03 units/minute) Associated with Adverse Events in Cardiac Surgery Patients?

14. Assessing the Extent of Fluoroquinolone-Cation Interactions at a Teaching Hospital

\section{Tuesday, February 7, 2012 • Mardi 7 février 2012}

1. A Systematic Analysis of Pharmacist Referrals Originating from the Order Desk

2. Audit of Antibiotic Duration of Therapy, Appropriateness and Outcome in Patients with Nosocomial Pneumonia Following the Removal of an Automatic Stop-date Policy

3. Identification of Predictors of Infection in Acute Burn Injury Patients

4. Impact of a Multi-Layered Initiative to Improve Venous Thromboembolism Prophylaxis Rates in Medicine Patients in a Community Hospital Setting 
5. A Pilot Study to Evaluate Methodology for Assessing the Treatment of Low Back Pain

6. Classifications of Drug Related Problems Discovered during Brown Bag Medication Reviews for Older Adults

7. Evaluation of Education on Preventive Care and Poison Control Center Awareness during Brown Bag Medication Reviews for Older Adults

8. Self-perceived Knowledge Gained and Satisfaction from Brown Bag Medication Reviews for Older Adults

9. Cost Analysis of an Insulin Pen Pilot Project in an In-Patient Hospital Setting

10. Venous Thromboembolism Prophylaxis Electronic Physician Risk Assessment Tool

11. Fingertip Sampling is a More Sensitive Evaluation of Aseptic Technique Competency

12. Potential Cross-reactivity between Prasugrel and Clopidogrel

13. Total Parenteral Nutrition: Supporting Practice through Standardized Solutions

14. Determination of Tobramycin Pharmacokinetics in Burn Patients to Evaluate the Potential Utility of Once Daily Dosing in this Population

\section{Wednesday, February 8, 2012 • Mercredi 8 février 2012}

1. Diagnostic Reasoning by Hospital Pharmacists: An Assessment of Attitudes, Knowledge, Skills, and Learning Needs

2. A Pilot Study on the Use of Warfarin Sliding Scales in Hemodialysis Patients

3. Potentially Inappropriate Medication Use in Elderly Patients Presenting to the Emergency Department

4. Intentional Overdose of Enoxaparin

5. Implementation of a Pharmacy Residency Program in Primary Care

6. Differences in Nova Scotian Pharmacists' Experiences and Barriers to Providing the Emergency Contraceptive Pill Based on Primary Practice Site

7. Chemotherapy-Induced Nausea and Vomiting in Children Receiving Ifosfamide plus Etoposide: Preliminary Results

8. Improving a Complex Pharmacist Scheduling Model at a Tertiary Care Hospital

9. Statin Treatment Use in Diabetics with Breast Cancer: A Potential C-Reactive Protein Mediated Benefit

The texts of poster abstracts are published exactly as submitted by the authors and have not undergone any copyediting by the Canadian Journal of Hospital Pharmacy. Le Journal canadien de la pharmacie hospitalière ña pas soumis le texte des résumés des affiches à une révision linguistique et les publie ici tels que remis par les auteurs.

\section{An Outpatient Parenteral Antimicrobial Therapy Program Experience at the University Health Network \\ Anjie Yang ${ }^{l,}$ Ron Fung', James Brunton', Linda Dresser ${ }^{1,3}$ \\ ${ }^{1}$ Department of Pharmacy, University Health Network, Toronto, ON \\ ${ }^{2}$ Department of Medicine, Division of Infectious Diseases, University Health Network, Toronto, ON \\ ${ }^{3}$ Leslie Dan Faculty of Pharmacy, University of Toronto, Toronto, ON}

Rationale for Report: Current literature shows that Outpatient Parenteral Antimicrobial Therapy (OPAT) programs improve treatment efficacy and safety for patients discharged on intravenous (IV) antimicrobials. However, such programs are still relatively new in Ontario. In response to cases of treatment related adverse events with the present standard of care, an OPAT program was developed, implemented and evaluated.

Description of Situation: Adverse events involving nephrotoxicity among outpatients receiving IV vancomycin identified a gap in antimicrobial management. The OPAT program was developed to address this gap.

Development of Service: This interprofessional antimicrobial stewardship effort was fully implemented in February 2010 and included all eligible patients discharged on IV antimicrobials. The OPAT pharmacist role is integral in selecting the choice of therapy and vascular access device, as well as providing patient education and monitoring throughout the treatment course.

Evaluation: A retrospective matched cohort study was conducted involving 108 surgery patients enrolled in the OPAT program between Feb 1 and Nov 30, 2010, with cure rates, 30-day rehospitalization within our hospital and length of stay as primary endpoints. The OPAT patients were matched 1:1 to historical controls discharged between Jan 1, 2001 and Jan 1, 2010 based on age, gender, type of surgery, infection, and co-morbidities (Charlson Co-morbidity Index). Due to the rigor of the matching criteria, twenty-one OPAT patients were matched. For this cohort, the OPAT program was trending towards improved cure rates $(61.9 \%$ vs $57.1 \%)$, reductions in rehospitalization ( $14.3 \%$ vs $28.6 \%)$ and mean length of stay (10.7 vs 13.9 days) compared to the control group.
Importance to Practice: The OPAT program is an innovative expansion of the antimicrobial stewardship program, with the pharmacist role being crucial in optimizing antimicrobial therapy in the outpatient setting. A further evaluation of the impact of this project on rehospitalizations province-wide is in progress.

\section{Antimicrobial Prescribing Practices for Catheter Urine Cultures}

Jonathan Chiu, William G Thompson, Thomas W Austin, Michael John, Zafar Hussain, Anne Marie Bombassaro, Sarah Connelly, Sameer Elsayed London Health Sciences Centre, London, ON

Rationale: To investigate the literature suggestion that positive catheter urine cultures frequently result in unnecessary antimicrobial prescribing. Objectives: The study objective was to determine the need for antimicrobial stewardship directed to catheter urine cultures by evaluating concordance of treatment decisions between an expert panel and prescribers.

Methods: This was a retrospective case-control study of adults at a tertiary care centre. Cases and controls were defined as having positive and negative catheter urine cultures, respectively, and were identified through a microbiology laboratory report. Medical records were consecutively screened to select a convenience sample of 80 inpatients ( 40 per group). Abstracted patient histories were independently evaluated by an expert panel (3 Infectious Diseases consultants) blinded to decisions of prescribers and fellow panelists. The primary endpoint of concordance was defined as agreement between experts and prescribers (majority response) with respect to the indication for therapy. Secondary endpoints including unnecessary days of therapy and predefined patient outcomes were assessed over a 7-day period.

Results: Target enrolment required screening 591 charts. Intensive care stay, immunosuppression and emergency/outpatient status were the main reasons for exclusion. Baseline demographics between groups were comparable. Concordance was 40\% (16/40) and 85\% (34/40) in cases and controls, respectively (OR 0.118, 95\% CI 0.04-0.344; $\mathrm{p}<0.0001$ ). The most common reason for discordance was the administration of 
antibiotics when not indicated, 23/24 cases versus 5/6 controls, and accounted for 165 and 32 unnecessary days of therapy per 1000 inpatient days, respectively (OR 6.04, 95\% CI 3.81-9.57; p<0.0001). Adverse effects occurred in $2 / 23$ case patients who received antibiotics when not indicated.

Conclusion: A significantly lower concordance was observed between treatment decisions of experts and prescribers in response to positive versus negative cultures. Educational initiatives should focus on appropriate treatment indications for catheter urine cultures to reduce unnecessary therapy and to minimize adverse effects.

\section{Description of Propofol Use in a Pediatric Intensive Care Unit \\ Hiromi Koriyama', Margaret L Ackman², Jonathan P Duff, Alice W Chan ${ }^{2}$ \\ ${ }^{1}$ Pharmacy Services, Covenant Health, Edmonton, $A B$ \\ ${ }^{2}$ Pharmacy Services, Alberta Health Services, Edmonton, $A B$ \\ ${ }^{3}$ Department of Pediatrics, University of Alberta, Edmonton, $A B$}

Rationale: Propofol infusions continue to be used in pediatric intensive care units (PICUs), despite being contraindicated in this population due to concerns of propofol related infusion syndrome (PRIS). However, some studies have not identified harm with propofol use in PICUs. The Canadian Pediatric Critical Care Network (CPCCN) prepared a consensus statement on propofol use in PICUs, suggesting a $4 \mathrm{mg} / \mathrm{kg} / \mathrm{h}$ maximum mean rate and 24 hour maximum duration. Since this statement was developed, no studies have evaluated propofol use in the Stollery Children's Hospital PICU.

Objectives: To describe the practice patterns of propofol use in a singlecenter PICU, the rate of concordance of propofol use with CPCCN guidelines, and assess for the presence of signs and symptoms of PRIS. Methods: A retrospective chart review of initial propofol infusions used in PICU patients admitted during January 1 - December 31, 2009 was conducted. Charts were reviewed for propofol infusion characteristics, and presence of signs and symptoms of PRIS.

Results: Data were collected from 223 patients. The mean of the average hourly propofol dose (including boluses) was $2.8 \pm 1.2 \mathrm{mg} / \mathrm{kg} / \mathrm{h}$ and the average infusion duration was $10.3 \pm 6.7$ hours. $86.5 \%$ and $97.8 \%$ of infusions were concordant with CPCCN guideline maximum mean rate and duration, respectively. No cases of PRIS or deaths associated with propofol infusions were identified.

Conclusions: Initial propofol infusions are being used in the Stollery Children's Hospital PICU with a mean average hourly dose of 2.8 $\mathrm{mg} / \mathrm{kg} / \mathrm{h}$ and average duration of 10.3 hours. The majority of use was in concordance with CPCCN guidelines, but the need for consideration of the use and contribution of bolus doses to hourly propofol dose was identified. The use of initial propofol infusions in PICU patients appears to be safe, as an association with presence of signs and symptoms of PRIS, cases of PRIS, or death were not found.

\section{Providing Comprehensive Assessment and Pharmaceutical Care at the Geriatric Clinic for Parkinson's \\ Greta Mah, Joyce Lee \\ North York General Hospital, Toronto, ON}

Rationale: Parkinson's disease (PD) is a common neurodegenerative disease with majority of patients being over the age of 60 . Traditionally, PD management focuses on only motor features which are more apparent. However, recent studies showed up to $60 \%$ of patients also suffer from non-motor symptoms (NMS) leading to cognitive, autonomic and neuropsychiatric dysfunction which result in greater disability and hospital admission. Unfortunately, NMS are often under-diagnosed. In addition, elderly patients with PD are at high risk of experiencing drug therapeutic problems (DTPs) from their complex medication regimen. Therefore, a Geriatric Clinic with a physician-pharmacist team was established to provide comprehensive assessment and pharmaceutical care.

Objectives and Descriptions: A prospective observational study was designed to determine whether comprehensive assessment could identify previously unrecognized NMS and to describe the nature of DTPs. Patients were seen by pharmacist followed by physician during each clinic appointment. Patients also have easy access to the pharmacist for telephone consultation or urgent assistance. All patients seen from Jan 2008 to Nov 2009 were included in the study which was approved by the ethic review board.

Results: One hundred and forty patients with an average age of 76 were assessed. About 4 DTPs per patient were identified. Most common DTP was NMS required treatment such as dementia, depression, anxiety, constipation, psychosis, orthostatic hypotension and sleep disorders. Other common DTPs were adverse drug reactions (ADRs), subtherapeutic dosing and medication non-adherence. Over 120 ADRs were identified. Most common presentations were hallucination, confusion, dizziness, sedation and impulsive behaviours (i.e. gambling, hypersexuality) which often lead to caregiver stress and E.R. visits if not identified early.

Conclusion: Patients and families appreciated the comprehensive assessment and pharmaceutical care provided by the clinic. Many NMS and DTPs were managed to maintain patient's function, quality of life and to prevent unnecessary hospital admission.

\section{Performance of Clinical Prediction Tools Used to Predict Major Bleeding in Patients Receiving Anticoagulation Therapy \\ Sarah Burgess, Alejandro Lazo-Langner, George Dresser, Richard Kim, Natalie Crown \\ London Health Sciences Centre, London, ON}

Rationale: Fear of bleeding is a major determinant of the underuse of anticoagulation therapy. Studies suggest clinical tools developed to predict major bleeding have poor agreement and predictive ability; however, guidelines recommend the HAS-BLED score to assess bleeding risk in patients with atrial fibrillation. The purpose of this study is to evaluate the performance and utility of these tools in a real-world clinical setting. Methods: A retrospective cohort study was performed at the anticoagulation clinic at University Hospital, London Health Sciences Centre. Clinic charts of patients receiving warfarin therapy between September 2008 and February 2011, were reviewed. Bleeding risk factors identified at first clinic visit were used to apply bleeding risk tools (Outpatient Bleeding Risk Index, Contemporary Bleeding Risk Model, HEMORR ${ }_{2}$ HAGES, HAS-BLED) which stratified major bleeding risk as low, moderate or high. The primary endpoint of agreement in risk stratification between the tools was assessed using Kendall's tau-b coefficient. All predefined major and clinically relevant non-major bleeding events were identified from hospital charts and emergency department encounters. The predictive ability of each tool was evaluated using the C-statistic as a secondary endpoint.

Results: The study included chart-abstracted data for 321 patients on warfarin therapy with a follow-up of 319 patient-years to assess for bleeding events. The stratification of bleeding risk was inconsistent between the tools, evidenced by Kendall's tau-b coefficients for agreement (0.30 to 0.54 ). There were 3.8 major bleeds and 8.1 clinically relevant non-major bleeds per 100 patient-years of observation. C-statistics for predicting major bleeding were $0.61,0.67,0.71,0.74$ for Outpatient Bleeding Risk Index, HAS-BLED, Contemporary Bleeding Risk Model, 
and HEMORR 2 HAGES, respectively, demonstrating limited predictive ability.

Conclusion: Bleeding risk prediction tools demonstrated poor agreement and limited predictive ability for major bleeding events. Further study should focus on how to incorporate such tools into clinical decision making.

\section{Development of a Clinical Institute Withdrawal Assessment (CIWA) Scale and Treatment Regimen for the Management of Alcohol Withdrawal \\ Rajini Retnasothie, Jason Volling \\ University Health Network, Toronto, ON \\ University of Toronto, Toronto, $O N$}

Rationale: The Clinical Institute Withdrawal Assessment Scale Alcohol revised (CIWA-Ar) with symptom-triggered benzodiazepine therapy is the management of choice for alcohol withdrawal. Preliminary exploration revealed that practice at the University Health Network (UHN) was not completely aligned with these recommendations.

Description: The objective of this project was to determine which factors UHN clinicians felt impeded the current alcohol withdrawal management process. The goal was to use this information to modify the CIWA-Ar tool and develop management regimens which integrate the best evidence and promote consistent, standardized use across our sites. Implementation: A focus group and survey were conducted to gather clinicians' opinions on barriers they faced when managing patients experiencing acute alcohol withdrawal. A working group was then formed to review survey results and current evidence, revise the CIWA scoring tool and develop management regimens.

Evaluation: Survey respondents identified inconsistencies in the prescribing process as well as variation in benzodiazepine choice, dose, route, and frequency as major impediments to optimal workflow. The previous CIWA scoring tool was deemed user-unfriendly, leading many assessors to use tools from other centers. Respondents also indicated that most benzodiazepine orders were not linked to CIWA scores. Based on this information and the evidence, the CIWA scoring tool was revised and standard orders were developed in paper and electronic form, for outpatients and inpatients, respectively. These orders feature three unique benzodiazepine regimens, selected based on patient factors, which correlate directly to CIWA scores.

Importance: By creating a more user-friendly CIWA-Ar tool with correlating benzodiazepine orders, we are promoting symptom-triggered therapy, which is proven to reduce the risk of mortality associated with alcohol withdrawal (when compared to fixed dosing therapy). The modified scoring tool and order sets can be used by other centers that are also facing challenges with their alcohol withdrawal management process.

\section{Implementation of an Insulin Pen Pilot Project in an In-Patient Hospital Setting \\ Sara Kynicos ${ }^{l}$, Jin Hyeun Huh ${ }^{1,2}$ \\ ${ }^{1}$ University Health Network, Toronto, ON \\ ${ }^{2}$ Leslie Dan Faculty of Pharmacy, University of Toronto, ON}

Rationale: Insulin is considered a high-risk medication due to its potential to cause severe patient harm when implicated in adverse drug events. Following insulin medication incidents in our institution, the Safe Medication Practice committee recommended trialling a novel approach; in-patient administration by nurses using insulin pens.

Objective: To implement an insulin pen pilot in general internal medicine (98 beds across 3 floors) at one hospital site over 3 months, then measure safety outcomes related to medication incidents and satisfaction outcomes using nurse and patient feedback.

Method: In order to ensure that nursing staff would be able to use the pen devices and that new types of errors would not be introduced, a human factors usability study was conducted. This simulated various scenarios to test all steps in the process; including loading cartridges, labelling, priming, using safety needles and administration of dose. The results of this testing were used to design the pilot workflow processes and education for staff. All existing formulary insulins were made available in pen devices as floor-stock and each patient was allocated their own pen for each insulin type.

Results: A review of all reported incidents showed that no medication incidents involving insulin were reported on the pilot floors. A survey of nursing staff showed they preferred to use the insulin pens with $75 \%$ wanting to continue. The staff felt that the pens decreased the risk of administration of incorrect doses, were easy and efficient to use and allowed for increased patient teaching opportunities. A patient survey showed that the majority of insulin users $(87 \%)$ preferred for nurses to administer their insulin with pen devices.

Conclusion: This project demonstrates that insulin pen devices can be successfully implemented in an in-patient hospital setting, and should be considered as an option to potentially reduce errors with this high-risk medication.

\section{Drug Use Evaluation of Intravenous Tranexamic Acid: Adherence to Hospital Guidelines and Adverse Events in an Off-label Use in Orthopedic Patients \\ C Dara \\ North York General Hospital, Toronto, ON}

Rationale: Routine administration of intravenous tranexamic acid (IVTA) for off-label use during total knee replacement in patients without history of thromboembolic disease is associated with a $67 \%$ reduction in red blood cell (RBC) transfusions and, in those transfused, with a reduction in the number of units administered. In the literature, IVTA treatment was not associated with an increase in thromboembolic complications.

Objectives: The primary objective was to evaluate utilization of IVTA based on Pharmacy and Therapeutics (P\&T) approved criteria in both knee and hip orthopedic patients. The secondary objective was to describe safety outcomes in patients treated with IVTA, after 6 months of usage

Study Design and Methods: The agent was approved for addition to North York General Hospital (NYGH) formulary in January 2011 at a dose of $10-15 \mathrm{mg} / \mathrm{kg} /$ dose given intra-operatively and repeated 3 hours post-operative. A retrospective drug use evaluation was completed on all patients identified through the pharmacy electronic database receiving IVTA between January - June 2011. Electronic charts were reviewed by a pharmacist using an audit tool. Criteria evaluated included compliance with prescribing criteria in orthopedic patients, incidence of hemoarthrosis, venous thromboembolism (VTE) and transfusion rates, and any hospital readmission.

Results of Study: From January 2011 to June 2011, 31 orthopedic patients received IVTA. 


\begin{tabular}{lccccc}
$\begin{array}{l}\text { NYGH approved } \\
\text { indications for IVTA }\end{array}$ & $\mathbf{n}$ & $\begin{array}{c}\text { RBC } \\
\text { Transfused }\end{array}$ & $\begin{array}{c}\text { Hemo- } \\
\text { arthrosis }\end{array}$ & VTE & $\begin{array}{c}\text { Readmit/ } \\
\text { ER visits }\end{array}$ \\
\hline Hip/Knee revision & 1 & 1 unit & 0 & 0 & 0 \\
\hline Major long bone fractures & 1 & 1 unit & 0 & 0 & 0 \\
\hline $\begin{array}{l}\text { Bilateral total knee } \\
\text { replacement }\end{array}$ & 26 & No & 0 & 0 & 0 \\
\hline $\begin{array}{l}\text { Single knee replacement } \\
\text { in an anemic patient }\end{array}$ & 3 & No & 0 & 0 & 0 \\
\begin{tabular}{l} 
Hgb $<125 \mathrm{~g} / \mathrm{L}$ \\
\hline
\end{tabular}
\end{tabular}

Conclusion: Use of tranexamic acid for the off-label indication to decrease need for blood transfusions in orthopedic surgery has been within the NYGH approved indications with no adverse events (hemoarthosis or VTE) complicating hospital stay, discharge or follow-up

\section{Improving Continuity of Care Post-Hospital Discharge: A Needs Assessment of Community Pharmacists \\ Minh-Hien Le, Vincent Teo \\ Sunnybrook Health Sciences Centre, Toronto, ON}

Rationale: Increasing information exchange between community and hospital pharmacists has been shown to increase the number of interventions by both. Sunnybrook Health Sciences Centre (SHSC) currently provides patients with a discharge summary (DS), generated by SHSC's e-discharge program, which outlines details of their hospital admission. Such information may be useful to community pharmacists to ensure continuity of care.

Description: To determine the importance of various hospital admission details to community pharmacists and to identify common barriers that are encountered when ensuring continuity of care.

Steps: A feedback survey was developed from findings of previous studies and information currently provided in the DS. Surveys were distributed to over 600 community pharmacists working within a $10 \mathrm{~km}$ radius of SHSC. The survey asked pharmacists to rank the importance of patient hospital admission details using a five-point scale. It also asked a series of open-ended questions relating to barriers in providing continuity of care, feedback on other discharge tools seen in practice, and other information that would be helpful in improving post-discharge care.

Evaluation: Sixty pharmacists responded and $90 \%$ or more ranked the following as being "very important": current medication list on discharge; list of discontinued medications; list of medications which have been adjusted. Details such as procedures performed while in hospital, results of procedures and tests performed in hospital, list of complications occuring while in hospital and course in hospital were ranked from "neutral" to "not important" by the majority of pharmacists. Common barriers included prescription processing issues, lack of contact information and medication reconciliation issues. Pharmacists commented that lab results might be helpful to facilitate patient monitoring, particularly if the scope of practice of pharmacists was to expand.

Relevance: Having identified the needs of community pharmacists, we can improve communication tools that will enhance the continuity of care for patients.

\section{Patient Recall of Interaction with a Pharmacist During Hospital Admission \\ Douglas Doucette, Regional Pharmacy Services; Carole Goodine, \\ Zone 3 Fredericton; Jodi Symes, Zone 2 Saint John; Erin Clarke, \\ Zone 1 Moncton; Scott Simpson, Zone 7 Miramichi \\ All authors affiliated with Pharmacy Services, Horizon Health Network, NB}

Purpose: CSHP 2015 Objective 1.5 proposes that at least $50 \%$ of recently hospitalized patients or their caregivers will recall speaking with a pharmacist while in the hospital. Following a major reorganization of health authorities in New Brunswick, we sought to determine the baseline prevalence of patients who recall interacting with the pharmacist during their hospital admission, and their level of satisfaction with these encounters.

Methods: Former inpatients from 27 units in 9 hospitals in Horizon Health Network were randomly selected to complete a telephone questionnaire within 5 to 7 months of discharge from hospital. Patient responses were validated against pharmacist documentation in the patient health record. Approval was granted by Horizon's Research Ethics Board. Funding was provided by Medbuy Incorporated.

Results: From August 2010 to July 2011, 1055 former inpatients were screened and 399 completed the telephone survey. Respondents were mainly female (56\%) with mean age 67 years. During their recent admission $46 \%(n=184)$ of patients recalled speaking with a pharmacist. Eighty-nine percent responded they were "satisfied" or "very satisfied" in their interaction with the pharmacist. If the hospital offered the opportunity to talk with a pharmacist who could help answer their questions about medications, $83 \%$ of respondents indicated they would want this service. For patients who recalled speaking with a pharmacist, a sample of 181 patients was explored for evidence of pharmacist intervention or encounter with subject (medication history, consultation, etc.). Pharmacist documentation was found in health records of $65 \%$ of patients who recalled an interaction and in $88 \%$ of all patients.

Conclusions: During a recent hospital admission, $46 \%$ of former inpatients recalled speaking with a pharmacist. The vast majority of patients were satisfied with this interaction and would welcome future services from hospital pharmacists.

\section{How Well Do Pharmacy Clinicians Perform in a Patient Simulation-Based Admission Medication Reconciliation Validation Program?

 Sara Ingram ${ }^{1,2}$, Shiwani Chhibbar ${ }^{1,3}$, Olavo Fernandes ${ }^{1,2}$ \\ ${ }^{1}$ University Health Network, Toronto, $O N$ \\ ${ }^{2}$ Leslie Dan Faculty of Pharmacy, University of Toronto, Toronto, ON \\ ${ }^{3}$ University of Waterloo School of Pharmacy, Kitchener, ON}

Rationale: Hospitals are looking for effective strategies to implement medication reconciliation, train clinicians, and meet accreditation standards. The purpose of this study was to assess performance of pharmacy clinicians participating in a standardized formal medication reconciliation training program and compare the results among participant groups.

Description: Pharmacy clinicians participated in an educational program designed to teach and evaluate key competencies linked to medication reconciliation. In the education phase, participants completed a pre-reading package and attended a group learning session that introduced medication reconciliation, best possible medication history $(\mathrm{BPMH})$ taking, and identification and coding of discrepancies. In the validation phase, participants interviewed a standardized patient and were evaluated on: 1) time to complete BPMH; 2) BPMH process accuracy; 3) identification and coding of discrepancies. Data was grouped by participant type and analyzed.

Evaluation: During a 5 year period, 135 pharmacy clinicians including pharmacists $(n=87)$, pharmacy students $(n=36)$ and pharmacy residents/interns $(n=12)$ participated in the program. In the validation phase, the overall mean BPMH score was $88.9 \%$ [range $64.0-100.0 \%$ ]. The mean BPMH scores for pharmacists, students and new graduates were $88.4 \%$ [56.0-100.0\%], 88.8\% [64.0-100.0\%] and 93.0\% [88.0- 
100.0\%] respectively. The overall mean BPMH completion time was 17 minutes [5-60] The average BPMH time (in minutes) by group was: pharmacists 20 [5-60], students15 [10-30] and residents/interns 17 [1223]. The overall discrepancy identification and coding accuracy scores were 94.4\% [50.0-100.0\%] and 99.5\% [90.0-100.0\%] respectively. The participants who used the BPMH interview guide $(102 / 135)$ had a higher average score than those that did not $(91.3 \%$ vs. $81.6 \%)$.

Importance: A standardized medication reconciliation validation process enables consistent teaching and promotes competence in BPMH taking and medication reconciliation. This process can be widely applied to other institutions and clinician groups. Notably, the evaluation scores are high and similar across pharmacy clinician groups.

\section{Who's Looking at the BPMH? Perception and Utilization of the Best Possible Medication History in a Tertiary Care Hospital \\ Sara Ingram ${ }^{1,2}$, Julia Streminsky ${ }^{1,2}$, Rosalyn Fleites ${ }^{1,3}$, Olavo Fernandes ${ }^{1,2}$ ${ }^{1}$ University Health Network, Toronto, ON \\ ${ }^{2}$ Leslie Dan Faculty of Pharmacy, University of Toronto, Toronto, ON ${ }^{3}$ University of Waterloo School of Pharmacy, Waterloo, ON}

Rationale: The Best Possible Medication History (BPMH) is a comprehensive medication history completed by clinicians, using several sources of information. The $\mathrm{BPMH}$ is used in medication reconciliation, where a systematic comparison with current orders helps ensure safe, accurate medication information transfer. The study hospitals had admission medication reconciliation completed for $>75 \%$ of inpatients, but it was unclear to what extent the BPMH was being used by healthcare providers. The primary objective of this study was to assess the utilization and integration into practice of the BPMH across healthcare professionals providing direct patient care at a tertiary hospital in order to better direct education and quality improvement measures.

Description: The study was conducted via a survey over a 10 -week period. Specific groups targeted included: Medicine, Nursing, Pharmacy and Allied Health. Participants were contacted by email via hospital mailing lists, or were provided a paper copy of the survey by study coordinators. Evaluation: This study $(\mathrm{N}=330)$ revealed the majority of respondents used the BPMH (64\%), of which $40 \%$ reported regular use (>10 times/week). An analysis of BPMH utilization by listed profession revealed that $50 \%$ of physicians, $63 \%$ of nurses, $70 \%$ of nursing leadership/nurse practitioners, $71 \%$ of dieticians and $93 \%$ of pharmacists use the BPMH. Participants who did not use the BPMH (36\%) cited being unaware of the resource as the most frequent reason for not using it. Further education on the BPMH, including its content and location is warranted to increase its clinical impact in patient care.

Importance: Findings suggest that the majority of clinicians were familiar with the BPMH and actively used it as part of routine patient care. However, results also highlight that more education about the purpose and availability of the BPMH is still required, in order for all clinicians to integrate it more commonly into their practice.

\section{The Evaluation of a New Hierarchical Teaching Model for Pharmacy Students in Experiential Education \\ Laura Tsang, Minh-Hien Le, Vincent Teo Sunnybrook Health Sciences Centre, Toronto, ON}

Rationale: Pharmacy students undergoing experiential education are traditionally mentored by pharmacist preceptors. The changing curriculum at the University of Toronto (UT) and increased number of pharmacy students have created stress on this system and other teaching models should be explored.
Description: To evaluate a new teaching model involving UT fourthyear pharmacy Structured Practical Experience Program (SPEP) students and residents mentoring junior pharmacy students under pharmacist supervision. The impact on learning, overall experience and pharmacist workload were examined.

Steps: Surveys were distributed to 13 second year UT pharmacy students completing their Early Hospital Experience (EHE) rotation and their mentors. Seven EHE students were matched with SPEP students, two with residents and four with pharmacists concurrently preceptoring SPEP students.

Evaluation: Of the nine EHE students with an SPEP/resident mentor, $89 \%$ found the experience worthwhile and $100 \%$ agreed this hierarchical teaching model should be repeated. All students agreed that a SPEP student/resident mentor enriched learning and those EHE students with pharmacist mentors wished they had this opportunity. For mentors, none "strongly agreed" to a significantly increased workload or impaired learning experience and described improved teaching skills and a worthwhile experience. Pharmacists agreed the program did not significantly increase workload, space constraints or disrupt SPEP learning and would coordinate this model again.

Relevance: This new hierarchical teaching model was well received. EHE students preferred SPEP/resident mentors as they were more accessible, familiar with the curriculum and could advise on course selection and career options. Traditionally, preceptoring students requires a large time commitment and many pharmacists feel they cannot grant the increasing requests for rotations. However, this study shows the successful implementation and feasibility of a student structured teaching model with supervision by pharmacists with both clinical and dispensing responsibilities. Furthermore, this model helps to maximize the number of students exposed to hospital pharmacy practice.

\section{Evaluation of the Effectiveness of Online versus Face to Face Interactions in Interprofessional Education \\ Vincent Ho, Joan Lee, Justin Lee Hamilton Health Sciences, Hamilton, $O N$}

Rationale for Report: The focus of interprofessional education (IPE) is the development of skills to facilitate collaboration but achieving this goal can be difficult due to logistical challenges. Utilizing online services may provide an effective and familiar way for students to interact.

Description of Concept: Undergraduate students from nursing, occupational therapy, pharmacy, physiotherapy and social work completed 2 clinical scenarios, one as an online interaction utilizing Google Docs, Gmail and Skype, then was compared to a face to face (F2F) interaction. Development and Implement of Project: Seven students were recruited and took part in the study. Two observers assessed the students on the number of pre-defined learning objectives achieved and the demonstration of interprofessional competencies in each interaction. Participants' experiences and attitudes toward IPE were assessed using pre- and post-interaction surveys and the Readiness for Interprofessional Learning Survey (RIPLS).

Evaluation of Project: The online and F2F interactions achieved 71\% and $86 \%$ of the learning objectives respectively. The F2F interaction resulted in a higher team score in the demonstration of interprofessional competencies and $57 \%$ of students also received higher individual scores. The F2F interaction was preferred overall by $71 \%$ of students and both observers. The F2F interaction was preferred by $71 \%$ of students in terms of logistics and $100 \%$ of students in terms of IPE experience and learning experience. RIPLS scores improved after completing the interactions. Participants highlighted components of each interaction that could be combined to improve future interactions. 
Concept's Importance and Usefulness to Current and or Future Practice: The online interaction may not be as effective as the F2F interaction in IPE. Students felt that the online interaction was less effective for discussion but may help address some logistical challenges. Educators should implement a combination of components highlighted by participants to help improve the online collaborative experience.

\section{Diabetes Education in Pharmacy Residencies across Canada \\ Henry Halapy, St Michael's Hospital, Toronto, ON \\ Gary Wong, University Health Network, Toronto, ON}

Rationale: Diabetes is a growing chronic health problem in Canada, requiring complex management. As a result teaching, pharmacy students about diabetes has become increasingly important. However, little is known about the amount and type of education regarding diabetes given to post-graduate pharmacy residents in Canada.

Objective: To evaluate the extent and type of training currently conducted around diabetes management in Canadian pharmacy residencies. Study Design and Methods: A web-based survey tool asking about exposure to diabetes topics (such as diabetes medication pharmacology, insulin dosing) in pharmacy residency training was distributed to pharmacy residency coordinators/ directors and residents across Canada via email. Most answers were recorded using a 5 point Likhert scale (no exposure, a little, some, a lot, resident becomes proficient). The survey was reviewed for face and content validity prior to distribution. Congestive heart failure, a frequently occurring chronic health problem in Canada, was used as a comparator therapeutic topic.

Results: A total of 41 responses were received (19 coordinators, 5 directors, 17 residents). Diabetes rotations were uncommon in Canadian pharmacy residencies ( $2.4 \%$ of responses), while cardiology rotations were common ( $81 \%$ of responses). Residents were exposed predominantly to no (4/15 topics, e.g., carbohydrate counting), a little $(5 / 15$ topics, e.g., blood glucose meters), or some (6/15 topics, e.g., sliding scales) with regard to diabetes topics while residents were exposed predominantly to some (8/15 topics, e.g., amiodarone use) or a lot (4/15 topics, e.g., blood pressure monitoring) to heart failure topics. Between $4-9 \%$ of residents became proficient in some heart failure topics (warfarin counseling) while very few residents became proficient in diabetes topics. Conclusions: This survey would suggest that pharmacy residents are exposed to diabetes topics relatively less than congestive heart failure. Greater exposure to diabetes topics may be beneficial in pharmacy residency training.

\section{Impact of a Drug Access Facilitator in an Outpatient Chemotherapy Clinic}

Mei Shi, Daniela Gallo-Hershberg, Nicole Harvey

North York General Hospital, Toronto, ON

Rationale: Obtaining reimbursement and access for treatment and supportive therapies for outpatient chemotherapy clinics has been fragmented where multiple stakeholders, including patients, are involved in the process. The role of a drug access facilitator (DAF) was investigated to reduce wait time to treatments, clinician administrative workload, and clinician and patient frustration.

Description and Steps Taken: Through interviewing key stakeholders, a needs assessment of the existing reimbursement process was conducted. This resulted in the development of a new streamlined workflow as well as drug specific access protocols, and led to the creation of the centralized $\mathrm{DAF}$ role. Because the DAF would require extensive knowledge in medications and experience in dealing with third-party payers, a pharmacy technician was selected to fulfill this role. A pharmacist was available for consultation for clinically challenging cases.
Evaluation: Over a 6-month period, the DAF managed a total of 140 reimbursement cases. The average time to approval for Exceptional Access Program applications was decreased from 35 days to less than 10 days with the DAF intervention. A similar pattern was observed with applications for the Special Access Program and Trillium Program. Also, centralization of the drug access workflow to a single individual reduced administrative workload for members of the interprofessional team. A follow-up written survey of clinic staff and patients reported high levels of satisfaction with the DAF with average scores of $90.8 \%$ and $88.3 \%$, respectively.

Importance: The DAF has demonstrated a positive impact for the chemotherapy clinic. This role can be applied to various medical specialities outside of oncology for any medication with outpatient reimbursement and access barriers. This concept also aligns with the future of pharmacy practice as it empowers pharmacy technicians to expand their scope of practice to promote seamless care.

\section{High Dose Methotrexate in Adult Oncology Patients: A Case-Control Study Assessing the Risk Association between Drug Interactions and Methotrexate Toxicity \\ April Chan, Irina Rajakumar \\ London Health Sciences Centre, London, ON}

Background: High-dose methotrexate, defined as dose $\geq 1 \mathrm{~g} / \mathrm{m}^{2}$, is commonly used in chemotherapy protocols. Certain drugs such as acyclovir, allopurinol, proton pump inhibitors (PPIs) and some antibiotics have been associated with delayed renal clearance of methotrexate and may predispose patients to toxicities. Currently, no specific recommendations exist on adjusting high-dose methotrexate regimen in the presence of potential interacting drugs. This study aims to determine whether presence of interacting drugs is associated with delayed methotrexate clearance.

Methods: This was a case-control study of adult oncology patients who received their first cycle of high-dose methotrexate. Cases were defined as patients who experienced delayed methotrexate clearance, as indicated by serum methotrexate level $\geq 0.1 \mathrm{umol} / \mathrm{L}$ at 72 hours. The primary endpoint was the frequency of interacting drugs between cases and controls. These were compared using Fisher's exact test. Where possible, adjustment for significant baseline differences was made using logistic regression. The secondary endpoint was frequency of methotrexaterelated clinical toxicities between groups and included myelosuppression, nephrotoxicity, hepatotoxicity and mucositis.

Results: From January 2004 to March 2011, 73 patients met study criteria, of which 23 were defined as cases. Significant baseline differences were high-dose methotrexate dose received and renal impairment. The presence of interacting drugs was not associated with delayed methotrexate clearance (OR 0.91, 95\% CI 0.24-3.38, p>0.999). After adjusting for high-dose methotrexate dose, drugs observed with the most frequency (allopurinol, PPIs and sulfamethoxazole/ trimethoprim) were not associated with delayed methotrexate clearance ( $\mathrm{p}$-values of $0.948,0.592$ and 0.204 respectively). Cases experienced more severe anemia (grade 2.52 versus $1.68, \mathrm{p}=0.007)$ and higher rates of mucositis $(65.2 \%$ versus $20.0 \%, \mathrm{p}<0.001)$.

Conclusion: This study showed no significant association between presence of interacting drugs and delayed methotrexate clearance. Patients who experienced delayed methotrexate clearance had more severe anemia and higher rates of mucositis. 


\section{Stability of Azacitidine Solutions in Sterile Water for Injection \\ Scott E Walker, Flay Charbonneau, Shirley Law, Craig Earle \\ Department of Pharmacy, Sunnybrook Health Sciences Centre; Odette Cancer Centre; Cancer Care Ontario; Ontario Institute for Cancer Research; University of Toronto; Toronto, $O N$}

Background: The azacitadine product monograph indicates that once reconstituted, it may be held for only 30 minutes at room temperature or 8 hours at $4 \mathrm{C}$. This results in significant wastage of this expensive drug, adding approximately $\$ 156,000$ to annual drug expenditures at our institution. The objective of this study was to evaluate the stability of azacitidine.

Methods: Vials of azacitidine were reconstituted with SWFI. For studies completed at $4 \mathrm{C}$ and $-20 \mathrm{C}$, the SWFI was at $4 \mathrm{C}$ at the time of reconstitution. Solutions of 10 and $25 \mathrm{mg} / \mathrm{mL}$ of azacitidine in syringes and vials were stored at either $-20 \mathrm{C}, 4^{\circ} \mathrm{C}$ or room temperature $\left(23^{\circ} \mathrm{C}\right)$. The concentration of azacitidine was determined by a validated, stabilityindicating liquid chromatographic method in serial samples over 9.6 hours at room temperature, over 4 days $4 \mathrm{C}$ and over 23 days at $-20 \mathrm{C}$. The recommended expiry date was determined based on the time to achieve $90 \%$ of the initial concentration using the fastest or lower limit of the $95 \%$ confidence interval of the observed degradation rate.

Results: Azacitidine degradation was observed to be very sensitive to temperature but not concentration $(10 \mathrm{mg} / \mathrm{mL}$ or $25 \mathrm{mg} / \mathrm{mL})$ or container (original glass vial or polypropylene syringe). Reconstitution of azacitidine with cold SWFI reduces degradation. At 23C, $15 \%$ of the initial concentration is lost after 9.6 hours, $32 \%$ lost after 4 days at $4 \mathrm{C}$ and less than 5\% loss was observed at -20C after 23 days.

Conclusions: More than $90 \%$ of the initial azacitidine concentration will be retained, with $97.5 \%$ confidence, if during the life of the product, storage at $23 \mathrm{C}$ does not exceed 2 hours, storage at $4 \mathrm{C}$ does not exceed 8 hours and storage at $-20 \mathrm{C}$ does not exceed 4 days. These expiry dates can significantly reduce wastage and cost where the time between patients does not exceed 4 days.

Key words: azacitidine, drug stability

In for a Penny, in for a Pound: Evaluation of Factors Affecting Learner Participation in the Pilot "Adapting Pharmacists' Skills and Approaches to Maximize Patients' Drug Therapy Effectiveness" E-learning Primary Health Care Program Caitlin Jones ${ }^{1,2}$, Barbara Farrell',, Ashley Gubbels ${ }^{1,2}$, Natalie Ward ${ }^{l, 4}$, Natalie Kennie ${ }^{5}$ Lisa Dolovich ${ }^{6}$, Derek Jorgenson' ${ }^{7}$, Brad Jennings, Pia Marks

${ }^{I}$ C T Lamont Primary Health Care Research Centre, Élisabeth Bruyère Research Institute, Ottawa, ON

${ }^{2}$ School of Pharmacy, University of Waterloo, Waterloo, ON ${ }^{3}$ Department of Family Medicine, University of Ottawa, Ottawa, ON ${ }^{4}$ Department of Anthropology and Sociology, University of Ottawa, Ottawa, ON

${ }^{5}$ Summerville Family Health Team, Mississanga, ON ${ }^{6}$ Department of Family Medicine, McMaster University, Hamilton, ON College of Pharmacy and Nutrition, University of Saskatchewan,

Saskatoon, $S K$

${ }^{8}$ Schulich School of Medicine \& Dentistry, The University of Western Ontario, London, ON

${ }^{9}$ Centre for Extended Learning, University of Waterloo, Waterloo, ON

Rationale and Objectives: The ADAPT (ADapting pharmacists' skills and Approaches to maximize Patients' drug Therapy effectiveness) e-learning program employs interactive e-learning, a new learning method for many pharmacists. In addition, the program requires regular participation in module activities and discussion to facilitate uptake of new skills in practice over a four month period. In this study, we sought to describe factors affecting learner participation in this e-learning primary health care program.

Study Design and Method: Qualitative and quantitative methods were employed. Learners estimated time commitment for each module. Extent of module participation and completion rates were analyzed using the Learning Management System Activity Log. Exit (for those withdrawing) and participation (for those remaining) surveys, composed of Likert scales and open-ended questions, were completed.

Results: During the pilot, 11/86 learners withdrew. Participation and exit survey completion rates were $26 / 75$ and $8 / 11$, respectively. $7 / 8$ who withdrew said they would take ADAPT again; most withdrew due to scheduling conflicts or other commitments. The participation survey showed a 50/50 split between those feeling satisfied and dissatisfied with their own participation. Many reported feeling discouraged occasionally and cited time, technical glitches and personal commitments as primary deterrents to full participation. There was variation with regard to how applicable learners felt specific content was to personal practice environment. Being better informed of program time and assignments in advance, were identified as facilitators to enhance planning for participation. Learners spent 9.5hrs (average) over two weeks on most modules. The evidence-based medicine module required $>15$ hrs over three weeks. Conclusion: While average weekly time spent on the ADAPT program was consistent with outlined requirements, participants struggled with technical aspects of online learning, as well as, committing time learning and practicing skills. Feedback will assist revisions to program marketing, 'readiness to participate' self-assessment and learner orientation.

\section{Moving Practice Forward with Early Exposure Students \\ Thomas E R Brown ${ }^{1,2}$, Farida Meghji ${ }^{1}$, Jane Mosley', Lisa McCarthy ${ }^{1,2}$ ${ }^{1}$ Women's College Hospital, Toronto ON \\ ${ }^{2}$ Leslie Dan Faculty of Pharmacy, University of Toronto, Toronto, ON Acknowledgements: Michaela Hogan, Adam Lam, Mitsumi Louis-Foster, Lauren Sule}

Rationale: Early practice experience rotations help students apply and build confidence with skills they are learning in the classroom while enhancing their appreciation for the aims of their education. Preceptors may feel challenged to identify project activities for these students given the limited amount of formal therapeutics education they have received. Description: Our aim was to demonstrate that early learners can make vital contributions to pharmacy department initiatives. Second-year undergraduate pharmacy students were assigned projects around optimal medication use and safety during 4-week rotations. Topics were strategically selected to lay the foundation for important safety initiatives at our institution, including: an audit of medication sampling practices; review of institutional practices around dangerous abbreviations; an audit of non-formulary medication requests; and a review of medication reconciliation initiatives in ambulatory care settings.

Evaluation: Four students participated in the program between May and August 2011. The audit of medication sampling found that $80 \%$ of samples in the audited areas were expired and has led to creation of a safer medication sampling pilot program in partnership with the Department of Psychiatry. Dangerous abbreviations have been removed from all pre-printed order sets and an online learning module has been created for staff. An assessment of non-formulary items in floor stock found that $20 \%$ of products in the hospital are non-formulary and raised questions about the concept of a formulary in an ambulatory institution. Lastly, the medication reconciliation review led to striking of a working group to develop a strategy for identifying patient populations to target in ambu- 
latory care thereby satisfying a test of compliance for a required organizational practice for Accreditation Canada.

Importance: This program demonstrates that learners early in their pharmacy careers can lay the groundwork for pharmacy initiatives around optimal medication use and safety.

\section{Provincial Implementation of Accreditation Canada Required Organizational Practices: The Alberta Experience \\ Dawn McDonald', Gwen Erdmann', Kefah Hayek', Nancy Louis', Jim Manley', Shaheen Nenshi Nathoo ${ }^{1}$, Laurine Sanderson ${ }^{2}$ \\ ${ }^{1}$ Medication Quality and Safety Team, Pharmacy Services, Alberta Health Services, Edmonton, $A B$ \\ ${ }^{2}$ Alberta Children's Hospital, Calgary, $A B$}

Rationale: Alberta Health Services (AHS) was created in 2008 through the amalgamation of nine former health regions and oversees 107 hospitals. The first AHS Accreditation Canada (AC) on-site survey was conducted in October 2010. Within the AC Managing Medications standards, seven Required Organizational Practices (ROPs) were identified as essential practices to be implemented. Four of these ROPs were within the purview of AHS Provincial Pharmacy Services: concentrated electrolytes, heparin safety, narcotics safety, and standardized concentrations. The challenge for the Provincial Pharmacy Services Medication Quality and Safety Team (MQST) was how to assess the current level of compliance at each site and fully implement the 4 ROPs across the province.

Description: Online assessments were created and used to quantify compliance with each ROP. The results of the assessments were used to develop a provincial implementation plan for each of the ROPs.

Steps Taken: The first online assessment, ROP Compliance AssessmentCurrent State, was completed in January 2011 by all 107 AHS acute care sites. This provided a provincial baseline. Alternatives were suggested to assist with product removal where required. When product removal was not possible, rationale was requested. A repeat assessment, Verification of Compliance, was completed in February 2011 to assess each site's progress in the actual implementation of the ROPs.

Evaluation: As of April 2011, the 4 ROPs within the Managing Medication standard were fully implemented throughout all 107 AHS sites. In September 2011, two pharmacy policies (High Potency Narcotics and Concentrated Electrolytes) were implemented provincially. A post-implementation audit will be conducted to evaluate safety mechanisms and processes in place, and determine the effectiveness of the Policies and Procedures.

Importance: Similar efforts to address AC ROP requirements provincially across 107 sites are unprecedented in Canada. Local engagement with provincial oversight was key to the success of this work.

\section{Drugs Obtained by Quebec University Health Centers through Health Canada's Special Access Programme - Descriptive Analysis by the programme de gestion thérapeutique des medicaments}

Élaine Pelletier ${ }^{l, 2}$, Benoît Cossette ${ }^{2,3}$, Céline Dupont, ${ }^{2,4}$, Nathalie


Paul Farand ${ }^{2,3}$, Daniel Froment, ${ }^{2,}$, Pierre Gaudreault,2, Raghu Rajan ${ }^{2,4}$ ${ }^{1}$ Centre hospitalier universitaire Sainte-Justine (CHU Sainte-Justine), Montréal, QC

${ }^{2}$ Programme de gestion thérapeutique des médicaments (PGTM), Québec ${ }^{3}$ Centre hospitalier universitaire de Sherbrooke (CHUS), Sherbrooke, QC ${ }^{4}$ Centre hospitalier universitaire de Santé Mc Gill (CUSM), Montréal, QC
${ }^{5}$ Centre hospitalier universitaire de Québec (CHUQ), Québec, QC ${ }^{6}$ Centre hospitalier universitaire de Montréal (CHUM), Montréal, QC

Rationale: University health centres (CHU) in the Province of Quebec commonly have to use medications authorized by Health Canada's Special Access Programme ("SAP-drugs"). Indeed many of their patients have diseases that are rare, complex or refractory to conventional drugs available on the Canadian market. In January 2008, Health Canada issued a new version of its guidance document defining principles and practices pertaining to SAP-drugs.

Objectives: To describe the overall utilization profile of SAP-drugs and the CHU's compliance with the administrative and therapeutic management guidelines proposed by Health Canada.

Design and Methods: Retrospective identification of SAP-drugs used between 1 April 2008 and 31 March 2009. Sampling procedure allowing to describe the main usage characteristics, including prescription frequency and costs, and measure compliance with five criteria for management and monitoring of use of SAP-drugs.

Results: The utilization profile brings to light the diversity, distribution, and impact of SAP-drugs use. For the reference one-year period, approximately 12,000 prescriptions were written, corresponding to more than 140 nonproprietary names. This represents $10.4 \mathrm{M}$ CAN\$ or $7.5 \%$ of the CHU's overall annual budget. Compliance with the criteria was very variable. Interesting qualitative observations and gaps have been identified at several stages of the drug distribution circuit.

Conclusion: Quebec's university health centres must improve their management of SAP-drugs, namely documentation and archiving, clinical monitoring, and funding.

\section{Safety and Efficacy of C $\mathrm{HOP}$ or R-CHOP for Treatment of Diffuse Large B-cell Lymphoma with Protease Inhibitor or Non-Protease Inhibitor Based Antiretroviral Regimens in HIV-Infected Patients: SCULPT Study}

Alison YJ Wong $g^{1,2,3}$, Suzanne Marcotte ${ }^{4}$, Mathieu Laroche $e^{4}$

Nancy L Sheehan ${ }^{3}$, Vishal Kukreti', Jean-Pierre Routy ${ }^{3}$,

Bernard Lemieux $x^{4}$, Jack Sekit, Danielle Rouleau ${ }^{4,5}$, Alice Tseng ${ }^{1,2}$

${ }^{1}$ University Health Network, Toronto, $\mathrm{ON}$

${ }^{2}$ University of Toronto, Toronto, $\mathrm{ON}$

${ }^{3} \mathrm{Mc}$ Gill University Health Centre, Montréal, QC

${ }^{4}$ Centre hospitalier de l'Université de Montréal, Montréal, QC

${ }^{5}$ Université de Montréal, Montréal, QC

Rationale: Use of combination antiretroviral therapy (cART) and cyclophosphamide, doxorubicin, vincristine, prednisone with or without rituximab $(\mathrm{CHOP}+/-\mathrm{R})$ for treatment of diffuse large B-cell lymphoma (DLBCL) substantially increases response rates but may also increase toxicity, possibly due to antiretroviral-antineoplastic drug interactions.

Objectives: We evaluated the frequency of confirmed or unconfirmed complete remission $(\mathrm{CR} / \mathrm{CRu})$ of DLBCL in HIV-positive patients treated with $\mathrm{CHOP}+/-\mathrm{R}$ while receiving protease inhibitor $(\mathrm{PI})$ versus non-PI based cART.

Design and Methods: A retrospective multi-centered observational pilot study was conducted in patients on cART, treated for DLBCL with CHOP+/-R between 2002-2010. Percentage of CR/CRu, 2-year overall survival (OS) and frequency of severe adverse events were evaluated. Possible predictors of CR/CRu between groups were evaluated by univariate logistic regressions.

Results: Thirty-four patients were included, $65 \%$ and 35\% of patients received a PI and non-PI based cART, respectively. Baseline characteristics were similar between both groups; $85 \%$ of patients were male, median age 43 years, $50 \%$ with International Prognostic Index (IPI) score 2-3, median 7 years since HIV diagnosis and $\mathrm{CD}^{+}$of $225 \mathrm{cells} / \mathrm{mm}^{3}$. 
$\mathrm{CR} / \mathrm{CRu}$ was achieved in $77 \%$ and $58 \%$ of patients in the PI and non-PI group, respectively $(\mathrm{p}=0.21$ ), with $65 \%$ and $63 \%$ of patients achieving 2-year overall survival $(\mathrm{p}=1.00)$. Univariate analyses showed that a lower IPI score and a higher total number of received chemotherapy cycles were significantly associated with higher CR/CRu rates ( $\mathrm{p}=0.02$ and 0.03 , respectively). Tolerability was similar between both groups except decreased frequency of anemia in the PI group $(23 \%$ versus $37 \%, \mathrm{p}=0.04)$.

Conclusion: Similar efficacy and tolerability of $\mathrm{CHOP}+/ \mathrm{R}$ was observed with PI based and non-PI based cART, though less anemia was observed in the PI group. Response rates appear to be higher in patients receiving a PI based cART but requires confirmation with larger studies.

\section{Drug Access Navigator Database: Improving the Management of Drug Access Cases through the Development and Implementation of a Computerized Database}

Allison Jocko, Janet Lui

The Scarborough Hospital, Toronto, $O N$

Most oral chemotherapy and related support medications prescribed for oncology patients in Ontario are not routinely funded by the provincial drug programs. Navigating patient access is challenging and requires special request letters, private insurance investigations, or enrolment in assistance programs. Tracking case status and associated workload is equally difficult. As referrals and complexities increase, the need for a tool to facilitate processes was identified. Consequently, a Drug Access Navigator Database (DAND) was created using Microsoft Access software to support the process of tracking cases and generating reports on workload and outcomes.

The DAND links to forms, websites, costs, and contact information. Reports capture number of referrals, time spent, time for case resolution by drug, monthly or per cycle patient cost savings for new cases, approval expiry list, and pending case list. In the month of August 2011, there were 49 referrals, and 33 hours spent with an estimated patient cost diverted of $\$ 130,000$.

After implementing DAND, tracking and managing drug access cases was found to be simpler and faster. It was also found to be an easy tool for workload tracking and solidifying the need for the role of a drug access navigator for Oncology drugs in Ontario

\section{Residents as Preceptors: Development, Implementation and Evaluation of a Pharmacy Summer Student Clinical Experience \\ Natalie Crown, Sarah Burgess, April Chan, Jonathan Chiu, Janice Lee, Kelly MacLean, Anne Marie Bombassaro \\ London Health Sciences Centre, London, ON}

Rationale: Hospital pharmacy residency programs seek to ensure graduates exhibit competencies in patient care and teaching. Opportunities for residents to precept students may be limited in the current program structure. Conversely, undergraduate pharmacy students are frequently employed in technical roles with limited opportunities to participate in patient care.

Description: A 1 week rotation was developed to provide residents with an opportunity to provide practice based teaching to pharmacy students in a patient care setting, and practice direct instruction, modeling and coaching skills. The rotation provided students the opportunity to practice curriculum based skills, enhance understanding of patient care roles for hospital pharmacists and the benefits of a residency.
Steps: The rotation took place during the clinical practice rotation in the final month of the residency program. Students were matched to clinical rotations based on interest, complexity and academic year completed. Resident and student specific objectives were developed. Participants completed a daily self-reflection guide and submitted an end of rotation evaluation in a blinded format.

Evaluation: Five residents and 5 students participated in rotations including critical care, cardiac care, general surgery, internal medicine and nephrology. All residents strongly agreed that the experience increased "readiness" to function as a preceptor in the future, with 4 of 5 strongly feeling that the experience provided the opportunity to develop teaching and precepting skills. All pharmacy students strongly agreed that the rotation enhanced understanding of clinical practice in a hospital setting and were influenced positively about a career in hospital pharmacy, with 4 of 5 being motivated to pursue a post-graduate residency.

Importance: There was unanimous agreement amongst participants that a clinical rotation conducted by pharmacy residents for undergraduate pharmacy students should be incorporated into the residency program. The opportunity was subjectively evaluated as benefiting both residents and students from teaching and learning perspectives, respectively.

\section{Imatinib Plasma Trough Concentrations and the Ability to Assess Patient Adherence \\ Andrea Narducci ${ }^{1}, S E$ Walker $^{1,2}$, C DeAngelis ${ }^{1,2}$ \\ ${ }^{1}$ Leslie Dan Faculty of Pharmacy, University of Toronto, Toronto, ON \\ ${ }^{2}$ Sunnybrook Health Sciences Centre, Toronto, ON}

Rationale: Imatinib mesylate is an oral tyrosine kinase inhibitor used for the treatment of chronic myeloid leukemia (CML) and gastrointestinal stromal tumours (GIST). Therapeutic drug monitoring has been recommended due to recent studies demonstrating improved clinical outcomes.

Objectives: To determine the proportion of patients with imatinib plasma trough concentrations (Cmin) below the therapeutic range and the probability that a sub-therapeutic Cmin level is due to patient non-adherence.

Methods: A literature search was conducted to identify adult pharmacokinetic (PK) studies that determined any of the following: imatinib clearance, volume of distribution or half-life. Data was pooled to calculate a weighted mean \pm SD for each PK parameter and these values were used to create a concentration-time profile based on a 1 compartment model. A Monte Carlo Simulation (MCS; Crystal Ball v.11.1.1.3) was then completed to generate a distribution of steady state Cmin values for 1 million patients. This distribution was based solely on kinetic data; however, patient adherence would also affect $\mathrm{Cmin}$. The MCS was used to demonstrate the probability of patient nonadherence (NA) according to the equation: NA $=100 \%-$ [The probability of a specific Cmin according to the distribution].

Results: The MCS demonstrated that at a standard dose of $400 \mathrm{mg}$ once daily, $63 \%$ of patients do not achieve the Cmin threshold (> 1000 $\mathrm{ng} / \mathrm{mL}$ ) recommended for CML patients, and $68 \%$ of patients are below $1100 \mathrm{ng} / \mathrm{mL}$, the target suggested for GIST patients. At Cmin levels between 0 and $300 \mathrm{ng} / \mathrm{ml}$, the probability of nonadherence is $90-100 \%$; whereas at Cmin levels greater than $800 \mathrm{ng} / \mathrm{ml}$, the probability of nonadherence is less than $50 \%$.

Conclusions: The majority of patients do not achieve the imatinib trough levels proposed for clinical efficacy with the standard dose of $400 \mathrm{mg}$ daily. Also, it is difficult to determine patient adherence based solely on Cmin levels. 


\section{Évolution de la thromboprophylaxie chez les usagers médicaux dans un institut universitaire Julie Méthot, Marie-Noëlle Bartholoméi, Karine Lejeune Institut universitaire de cardiologie et de pneumologie de Québec, Québec, QC}

La thromboprophylaxie s'inscrit dans une politique de sécurisation et d'amélioration de la prise en charge des patients. Cette dernière se place au cœur même de la prévention du risque thromboembolique chez les patients nécessitant des soins médicaux entraînant une immobilisation prolongée ou subissant une chirurgie. Des actions stratégiques ont été mises en œuvre au sein de l'Institut universitaire de cardiologie et de pneumologie du Québec afin de promouvoir et évaluer la thromboprophylaxie chez les patients médicaux admis. En 2008 la création et la mise en place d'un aide-mémoire sur la thromboprophylaxie fut placé avec les documents d'admission à l'urgence. L'aide-mémoire comprenait les indications, les contre-indications et les posologies des médicaments utilisés en thromboprophylaxie. Limpact de la création de cet outil a été évalué suite à la révision de 263 dossiers. Le taux de thromboprophylaxie est demeuré stable (36,5\% avant la mise en place de l'aide-mémoire à 38,7\% après l'implantation de l'aide-mémoire). Cette première étude nous a amené à reconsidérer et remanier notre approche de la prévention des complications thromboemboliques chez les patients médicaux admis. Létape suivante consista en la réalisation d'une ordonnance pré-imprimée de thromboprophylaxie. Elle fut annexée aux feuilles d'admission de l'urgence de l'établissement pour favoriser son utilisation et fut implantée en février 2010. Une révision de 241 dossiers a été faite. Nos données suggèrent un taux de thromboprophylaxie d'environ $72 \%$ chez les patients médicaux admis après l'implantation de cette prescription. Les actions posées et les évaluations effectuées ont permis de promouvoir et améliorer la prescription adéquate de thromboprophylaxie.

\section{0-2011 Perspectives on Drug Shortages in Hospitals in Quebec \\ $G$ Ottino $^{I}$, D Lebel L J J F Bssières $^{l, 2}$ \\ ${ }^{1}$ Pharmacy Practice Research Unit, Pharmacy Department, \\ CHU Sainte-Justine, Montréal, QC \\ ${ }^{2}$ Faculty of Pharmacy, Université de Montréal, Montréal, QC}

Rationale: Many stakeholders underscore the importance of the drug shortage problem and its risks, but few quantitative data have been published on the subject.

Objectives: To report all the drug shortages experienced in Quebec hospitals.

Study Design and Methods: This is a retrospective study based on all drug shortages reported by the wholesaler and individuals members of the Group Purchasing Organization (SigmaSanté) for Montreal, Laval and Eastern township regions for 12 months (e.g. August 30, 2010 to August 23, 2011).

Results: A total of 429 drug shortages were reported in 2010-2011 vs. similar 12-month period; 493 in 2006, 400 in 2007, 442 in 2008, 680 in 2009 and 385 in 2010 (only the 8 first months). The average duration of the drug shortages remained similar, with $103 \pm 85$ days [8-363] in 2010 vs $108 \pm 130$ days [5-1623] in 2006-2010. A smaller number of manufacturers involved in drug shortages was also noted (41 in 20102011 vs. 70 in 2006-2010), a decrease that was undoubtedly related to mergers and the integration or closing of certain drug manufacturers. Most of the reported shortages in 2010-2011 came from generic drug manufacturers and their relative ranking remained similar (i.e., in decreasing order, in 2006-2010, Apotex (19\% of total drug shortages), Pharmascience (14\%), Novopharm (now Teva) (12\%), Sandoz (10\%), Hospira (6\%), Teva (4\%), Baxter (3\%), Ratiopharm (taken over by Teva) (3\%), Omega (2\%), Taro (2\%) vs. in 2010-2011, Teva (23\%), Apotex
(16\%), Pharmascience (12\%), Hospira (7\%), Sandoz (7\%), Baxter (5\%), AA Pharma (created from the resale of certain licenses held by Apotex) (3\%), Schering (3\%), Abbott (3\%), Merck Canada (2\%). Most therapeutic classes were involved in drug shortages in 2006-2010 as in 2010-2011,

Conclusion: There are an important amount of drug shortages in Quebec hospital since 2006 and there are no sign of reduction of their occurrence.

\section{Multicenter Study on Environmental Contamination by Hazardous Drugs in Quebec Healthcare Centers \\ J-F Bussières ${ }^{l, 2}, K$ Touzin ${ }^{l}, C$ Tanguay ${ }^{l}$, E Langlois ${ }^{3}$, A Métra ${ }^{4}, M$ Lefebvre $^{3}$ ${ }^{1}$ Pharmacy Department and Pharmacy Practice Research Unit, CHU Sainte-Justine, Montréal, QC \\ ${ }^{2}$ Faculty of Pharmacy, University of Montreal, Montréal, QC \\ ${ }^{3}$ Centre de toxicologie du Québec, Institut national de santé publique du Québec, Québec, QC \\ ${ }^{4}$ Association paritaire pour la santé et la sécurité du travail - secteur affaires sociales, Montréal, QC}

Rationale: Many occupational exposure studies have revealed the presence of hazardous drugs contamination on work surfaces used for the preparation, storing and administration of hazardous drugs in healthcare centers. Most studies were conducted in the United States, Europe and Japan. This multicenter study is the first to be conducted in Quebec healthcare centers.

Objectives: To describe environmental contamination by hazardous drugs in Quebec healthcare centers.

Study Design and Methods: Descriptive, prospective, comparative study. Six standardized sites in the pharmacy and six sites on wards were sampled in each participating center. Samples were analysed for the presence of cyclophosphamide, ifosfamide and methotrexate by UPLCMS-MS. A sample was considered positive if these drugs were detected and a sample was considered contaminated if the detected concentration was above $1 \mathrm{ng} / \mathrm{cm}^{2}$.

Results: Twenty-five of 68 (37\%) Quebec healthcare centers were included in the study. A total of 259 samples were collected between April 2008 and January 2010. Each center had at least one positive sample for one of the three drugs tested (median [min-max] 5[1-12] positive samples per center and 0[0-3] contaminated samples per center). Overall, $135(52 \%)$ samples were positive for cyclophosphamide, $45(17 \%)$ were positive for ifosfamide and $3(1 \%)$ were positive for methotrexate. The pharmacy hood front grille was the sample site with the higher proportion of positive samples, with 23 out of 25 (92\%) samples positive for cyclophosphamide. The median [min-max] concentration of hazardous drug was of $0.004[0-28.0] \mathrm{ng} / \mathrm{cm}^{2}$ for cyclophosphamide, $\mathrm{ND}[0-8.6] \mathrm{ng} / \mathrm{cm}^{2}$ for ifosfamide and ND[0-0.58] $\mathrm{ng} / \mathrm{cm}^{2}$ for methotrexate.

Conclusion: Although hazardous drug traces are frequently found, a median of less than one sampling site per center was actually contaminated. Periodic surface contamination measurements are necessary to ensure that current practices limit occupational exposure to hazardous drugs.

\section{Temps associé à la purge des tubulures lors de la préparation d'une dose de médicament dangereux et contamination visuelle}

$M$ Voisine $^{l}, K$ Touzin $^{1}, R$ Therrien, J-F Bussières ${ }^{1,2}$

${ }^{1}$ Unité de recherche en pratique pharmaceutique, Département de pharmacie, CHU Sainte-Justine, Montréal, QC

${ }^{2}$ Faculté de pharmacie, Université de Montréal, Montréal, QC 
Justification : L'amorçage des sacs de médicaments dangereux ainsi que la purge centralisée à la pharmacie de la tubulure peuvent contribuer à minimiser l'exposition professionnelle aux médicaments dangereux. L'impact de cette centralisation sur la charge de travail est méconnu.

Objectifs : Évaluer l'impact de la purge centralisée des tubulures sur le temps associé à la préparation d'une dose de médicament dangereux et sur la présence de contamination visuelle à la pharmacie et à l'unité de soins d'hématologie-oncologie.

Méthodologie : Il s'agit d'une étude observationnelle comparative en deux phases. La première consistait à comparer le temps médian pour la préparation d'une dose de médicament dangereux par un assistanttechnique en pharmacie sans purge et avec purge des tubulures à la pharmacie. La seconde visait à évaluer le temps médian nécessaire à la préparation d'une dose de médicament dangereux par le personnel infirmier à l'étage, suite à la purge des tubulures par le personnel de la pharmacie. Pour l'étude, le médicament dangereux était remplacé par une solution d'eau stérile colorée (essence de cerise). La contamination visuelle était évaluée dans les deux phases.

Résultats : Dans la première phase, le temps médian [intervalle] de préparation d'un sac de médicament non purgé $(\mathrm{n}=30)$ était de 98 [80-167] secondes et le temps médian de préparation d'un sac purgé par la pharmacie $(\mathrm{n}=30)$ s'élevait à 295[207-520] secondes (Kruskal-Wallis $\mathrm{p}<0,0001$ ). Pour la deuxième phase, le temps médian requis pour la purge de la tubulure d'un sac par le personnel infirmier à l'étage $(\mathrm{n}=20)$ était de 60[50-95] secondes. Aucune trace de contamination visuelle n'a été observée pour ces deux phases de l'étude.

Conclusion : La centralisation de l'amorçage et de la purge de sacs de médicaments dangereux à la pharmacie triple le temps médian requis par le personnel de la pharmacie. D'autres études sont nécessaires afin de confirmer la réduction des risques d'exposition liés à cette centralisation.

\section{Is Vasopressin (> 0.03 units/minute) Associated with Adverse Events in Cardiac Surgery Patients? Hina Ahmed, Marcin Wasowicz, Lior Flor, Vivek Rao \\ Toronto General Hospital, Toronto, ON}

Rationale: Vasopressin is used in the setting of vasopressor resistant vasodilatory shock. The Surviving Sepsis guidelines recommend maximum vasopressin infusion of 0.03 units/minute as an adjunct to norepinephrine. Vasopressin has been used to help reduce norepinephrine dose and to prevent adverse events related to high dose norepinephrine. In higher doses vasopressin has been associated with adverse events including reduced cardiac output, decreased oxygen delivery, decrease in mixed-venous oxygen saturation leading to impaired tissue perfusion, reduction in platelet count, hyponatremia, and injury to splanchnic organs. The safety of vasopressin use in cardiac surgery patients has not been studied as a primary endpoint.

Objectives: Use of high dose vasopressin ( $>0.03$ units/minute) is associated with adverse events in cardiac surgery patients.

Study Design and Methods: Upon REB approval, retrospective chart review was conduced to identify patients scheduled for cardiac surgery receiving vasopressin (>0.03units/minute). Data was collected through retrospective chart review. Primary outcome: adverse events potentially related to high dose vasopressin. Secondary objective: review vasopressin infusion use in relation to other vaopressor support.

Results: Over a 60-day period, 280 patients underwent cardiac surgery and $34(12.5 \%)$ received vasopressin. Major perioperative bleeding was seen in 8 patients (26\%) receiving vasopressin. Vasopressin was infused at doses higher than recommended, ranging from 0.03 units/min -0.13 units/min with an average duration of 34 hours. Ischemic complications were seen in less than $10 \%$ of patients and only one patient developed bowel ischemia. Among all patients, $85 \%$ received norepinephrine concurrently. Norepinephrine was used first line in over $50 \%$ of cases. In majority (87\%) vasopressin was used an adjunct to other vasopressors. Average hospital LOS was 16 days as opposed to 6 days.

Conclusion: Adverse events were seen infrequently with high dose vasopressin. Due to limitations of this study, a prospective study will help capture the true incidence of adverse events.

\section{Assessing the Extent of Fluoroquinolone-Cation Interactions at a Teaching Hospital}

Wenya Miao, Pharmacy Student, University of Toronto, Toronto, ON

Rosemary Zvonar, The Ottawa Hospital, Ottawa, ON

Rationale: VBoth fluoroquinolone antibiotics (FQ) and divalent or trivalent cationic drugs (DTC), such as aluminum, magnesium, iron, calcium and zinc, are frequently prescribed in hospitalized patients. The interaction between oral FQs and DTCs is well recognized, and therapeutic failures due to impaired FQ absorption have been reported when these agents are taken concomitantly.

Objective: To determine the prevalence of the interaction between FQs and DTCs at our institution.

Methods: On a single day in July 2010, patients prescribed formulary oral FQs (levofloxacin and ciprofloxacin) and DTCs were identified by the Pharmacy computer system. The Medication Administration Records of these patients were examined and scheduled drug administration times recorded. An interaction was defined as follows: for levofloxacin, an administration time for the DTC within 2 hours before or after that of levofloxacin; for ciprofloxacin, an administration time for the DTC within 4 hours before or 2 hours after any ciprofloxacin dose. Based on the results of the initial review, additional interventions to those already in place to minimize the chance of interaction were implemented. A similar assessment was repeated in June 2011.

Results:

\begin{tabular}{lcc} 
& \multicolumn{2}{c}{ Review performed } \\
July 26, 2010 & Review performed & June 15, 2011 \\
\hline Number of patients on oral FQ & 61 & 68 \\
\hline $\begin{array}{l}\text { Number of patients on an oral FQ } \\
\text { and DTC }\end{array}$ & 27 & 38 \\
\hline Number of Interactions & 16 & 18 \\
\hline $\begin{array}{l}\text { Percentage of patients on both oral } \\
\text { FQ and DTC with interaction }\end{array}$ & $59.2 \%$ & $47.4 \%$ \\
\hline
\end{tabular}

Conclusion: We identified a significant proportion of patients receiving oral FQs and DTCs either concomitantly or with inappropriate spacing, despite education and additional computer warnings following the initial review. Healthcare workers must remain vigilant for this frequent interaction. Continual review and intervention may be required to limit its occurrence.

\section{A Systematic Analysis of Pharmacist Referrals Originating from the Order Desk \\ Danette Beechinor \\ Credit Valley Hospital, Mississanga, ON \\ University of Waterloo, Kitchener, ON}

Rationale for Report: Significant clinical pharmacist time is spent resolving problems which originate on the order desk, which is typical for many hospitals. There is no published or local data which describes the problems returned to the clinical pharmacist.

Description of Concept, Service, Role or Situation: A study of the types of problems which are returned to the floor pharmacist was undertaken to determine potential time saving policies and procedures which could be developed while ensuring patient safety and minimizing distribution related delays. 
Steps Taken to Identify and Resolve Problem: Two hundred forty documents (convenience sample) which were identified by the order desk pharmacist as needing follow up by the floor pharmacist were analyzed using content analysis methodology. The documents were categorized according to problem type and analyzed to determine if improved policies or programs could facilitate problem resolution by the order desk and serve patients more efficiently and safely. All categories were considered for potential time savings and safety enhancement. Examples of categories which emerged included: identifying patients' own nonformulary medication, inappropriate dosage form ordered crushed, confirm date of weekly and monthly medications and dosage adjustment for renal dysfunction.

Evaluation of Project: Hospital and departmental policies are under development as a result of this study to save time and enhance patient safety. Examples of policies include: pharmacist adjustment of antibiotics for poor renal function, non-formulary medication identification and documentation by technician during Best Possible Medication History, and standardized administration dates for weekly or monthly medications such as bisphosphonates.

Importance and Usefulness: A systematic exploration of the departmental needs and typical problems which arise on order entry was used to propose policy changes to improve efficiency in order entry. Systematic problem review by hospital pharmacies can identify time- and costsaving measures which enhance patient safety and delivery of care.

\section{Audit of Antibiotic Duration of Therapy, Appropriateness and Outcome in Patients with Nosocomial Pneumonia Following the Removal of an Automatic Stop-date Policy}

Jennifer Do, Sandra A N Walker, Scott E Walker, William Cornish, Andrew E Simor

Sunnybrook Health Sciences Centre. Toronto, ON

Rationale: Although automatic stop-orders (ASOs) may discourage inappropriately prolonged antibiotic therapy, risks to patient safety associated with premature antibiotic discontinuation resulting from ASO policies have been reported. An ASO policy necessitating reordering of antibiotics after day seven of therapy had been in place at our institution prior to 2002; but was revoked after instances of compromised patient care associated with inadvertent and inappropriate interruption of antimicrobial treatment. Comparative data of patient outcomes with and without institutional ASO policies is lacking.

Objectives: The study objective was to evaluate the impact of revoking the ASO policy on duration of antibiotic therapy, infection-related outcome, relapsing infection, occurrence of resistant bacteria and superinfection in patients with nosocomial pneumonia.

Study Design and Methods: A retrospective chart review of hospitalized adults (> 18 years) who developed nosocomial pneumonia requiring antibiotic therapy was conducted. Duration of antibiotic therapy, infection-related outcome (cure vs. failure), rate of relapsing infection, resistance and superinfection were compared for the pre and post-ASO policy revocation cohorts. An unpaired t-test or Fisher's exact test was used for interval and nominal data comparisons, respectively. Multiple regression analysis and propensity score adjustment were completed to adjust for imbalances between groups. A p-value $<0.05$ was considered significant.

Results: Forty-two eligible adults with nosocomial pneumonia per cohort were included. Duration of antibiotic therapy was not significantly different between cohorts (pre: $11.4 \pm 3.8$ days; post: $10.8 \pm 4.1$ days; $\mathrm{p}=0.43$ ). There were also no significant differences between the cohorts with regard to infection-related outcome (cure vs. failure), relapsing infection, or the occurrence of resistant bacteria or superinfection $(\mathrm{p}>0.5)$.
Conclusion: Revocation of the ASO policy for antibiotics at our institution was not associated with a longer duration of antibiotic therapy, or an increased incidence of infection-related mortality, relapsing infection, resistant bacteria or superinfection for patients with nosocomial pneumonia.

\section{Identification of Predictors of Infection in Acute Burn Injury Patients}

Laura Schultz, Sandra A N Walker, Marion Elligsen, Scott E Walker, Andrew Simor, Samira Mubareka, Nick Daneman

Sunnybroook Health Sciences Centre, Toronto, ON

Rationale: Burn patients are at high risk for infections; however, common indicators of infection are unreliable in this population and can lead to unnecessary use of antibiotics.

Objectives: The objective of this study was to determine if predictors of infection in adult acute burn injury patients could be identified in order to provide clinicians with a practical tool to aid in the diagnosis of infection in acute burn injury, thereby minimizing unnecessary antimicrobial exposure.

Study Design and Methods: A retrospective chart review was conducted on all adult acute burn injury patients admitted to the burn centre at SHSC over a 1 year period. If a patient was started on the antibiotics during the first 10 days of admission, they were assessed for infection using the American Burn Association guidelines. Patient demographic data and clinical parameters were collected from admission to day 10 of hospitalization. If the patient was started on antibiotics, information was gathered inclusively from 3 days before to 7 days after antibiotic initiation. Patients without infection were compared to patients with sepsis and those with infection using unpaired t-test, Fisher's exact, regression analysis and CART analysis.

Results: Of the 111 patients studied, 50\% had infection and 16\% had sepsis within the first 10 days of admission. Using regression analysis and CART identified breakpoints, it was determined that heart rate $\geq 110$ bpm, systolic blood pressure $\leq 100 \mathrm{mmHg}$ and intubation were the best predictors of sepsis; and fraction of inhaled oxygen $>25 \%$ and maximum temperature $\geq 39^{\circ} \mathrm{C}$ were the best predictors of infection $(p<0.05)$. The positive predictive value when all criteria were satisfied for both sepsis and infection was $100 \%$.

Conclusions: This pilot project identified significant predictors of infection and sepsis in acute burn injury patients. These predictors will be validated in a prospective study.

\section{Impact of a Multi-Layered Initiative to Improve Venous Thromboembolism Prophylaxis Rates in Medicine Patients in a Community Hospital Setting Shelita Dattani Queensway Carleton Hospital, Ottawa, ON}

Rationale: VTE Prophylaxis should be considered in almost every hospitalized patient and is a required organizational practice (ROP) for Accreditation Canada in 2011. We previously demonstrated that thromboprophylaxis is well embedded in our surgical population, however we anticipated underutilization of thromboprophylaxis in medicine patients. As part of a broader hospital review to prepare for this ROP, we evaluated thromboprophylaxis in our medicine population.

Description: Our multidisciplinary task group developed a one year plan to achieve compliance with the ROP. Our overall objective was that at least $85 \%$ of eligible patients would receive an order for thromboprophylaxis by September 30, 2011. This review describes efforts to achieve targets in medicine patients within the same period. 


\section{Steps Taken To Implement Change:}

Table 1: Measures and Improvement Strategies

Initiative Date Details

Baseline Audit January 2011 Validated assumptions about underutilization of thromboprophylaxis in medicineconfirmed need for intervention

\begin{tabular}{lll}
\hline $\begin{array}{l}\text { Education } \\
\text { Intervention }\end{array}$ & $\begin{array}{l}\text { February - } \\
\text { June 2011 }\end{array}$ & Multidisciplinary education initiative \\
\hline Second Audit & June 2011 & Evaluated impact of education \\
\hline PPO & & $\begin{array}{l}\text { Developed Emergency Department (ED) } \\
\text { Admission Preprinted Order (PPO - details } \\
\text { Intervention }\end{array}$ \\
& & $\begin{array}{l}\text { Pescribed elsewhere) with TP embedded. } \\
\text { with documentation and reminder for daily } \\
\text { reassessment appearing on medication } \\
\text { administration record }\end{array}$ \\
\hline Third Audit & August 2011 & $\begin{array}{l}\text { Evaluated impact of ED PPO on sample of } \\
\text { medicine patients - reviewed thrombo- } \\
\end{array}$ \\
& & prophylaxis at admission and audit day \\
\hline
\end{tabular}

Evaluation: Proportions were used to describe results presented below. The percentage of patients with appropriate prophylaxis was calculated as the number of patients in whom prophylaxis was indicated who received appropriate thromboprophylaxis.

Table 2: Results of Three Part Audit

(n represents total number of patients in whom prophylaxis was indicated)

Evaluation

Medicine Unit 1 Medicine Unit 2 (\% of Patients (\% of Patients with Appropriate with Appropriate Prophylaxis) Prophylaxis)

\begin{tabular}{lll}
\hline Baseline Audit $(\mathrm{n}=53)$ & 61 & 84 \\
\hline Second Audit $(\mathrm{n}=57)$ & 75 & 79
\end{tabular}

Third Audit

Pre PPO Intervention -

Thromboprophylaxis Rates On

Admission ( $\mathrm{n}=37$ )

Pre PPO Intervention -

Thromboprophylaxis Rates on

Day of Audit $(\mathrm{n}=37)$

Post PPO Intervention -

Thromboprophylaxis Rates on

Admission ( $\mathrm{n}=37)$

Post PPO intervention-

Thromboprophylaxis Rates on Day of Audit ( $\mathrm{n}=37)$

Relevance to Current and Future Practice: Our initiative supports that multifaceted interdisciplinary interventions were effective in achieving targets in medicine patients. We will continue to evaluate the use of this approach in other populations at our institution.

\footnotetext{
A Pilot Study to Evaluate Methodology for Assessing the Treatment of Low Back Pain Jerrold Petrofsky', Lee Berk ${ }^{l, 2}$, Gurinder Bains ${ }^{l}$, Geraldine Doyle, Shijie Chen', Lisa Baird', Paul Desjardins', Jill Stark ${ }^{3}$ ${ }^{1}$ Department of Physical Therapy, ${ }^{2}$ Department of Pathology \& Human Anatomy, Loma Linda University, Loma Linda, CA ${ }^{3}$ Pfizer Consumer Healthcare, Madison, NJ
}

Rationale: Low back pain (LBP) is a common and costly medical problem. Using a stopwatch to measure the time of the onset of the analgesic effect has not been evaluated in a LBP model.

Objective: This pilot study was to evaluate the sensitivity of using the methodology for assessing LBP relief with ThermaCare LowBack/Hip Heatwraps.

Study Design and Methods: Subjects with baseline primary muscle acute LBP were randomized to ThermaCare, oral placebo, unheated "sham" wrap, or oral ibuprofen. The last 2 treatments were included for blinding purposes only. Subjects stopped the first stopwatch upon feeling the "first perceptible" pain relief, and the second stopwatch upon feeling "meaningful" pain relief. Subjects also assessed pain relief hourly.

Results: Sixty-one subjects participated in this single-center, single-dose (single wrap wear occasion), randomized, single-blind, placebocontrolled trial. The TOTPAR 0-8 was significantly higher for ThermaCare Heatwrap than placebo group ( 22.0 vs. $11.5 ; \mathrm{p}<0.001)$. The mean pain relief scores over time for the Heatwrap group were significantly higher than the oral placebo at all time points. The time to confirmed first perceptible relief and time to meaningful pain relief were significantly shorter for ThermaCare compared to oral placebo (median minutes 96.5 vs. $>240$ and 215.7 vs. $>240$, respectively; $p<0.05$ for both comparisons). Among subjects who received ThermaCare, 53.8\% reported both first perceptible and meaningful relief, compared to $28 \%$ in oral placebo. Three mild adverse events were reported; the investigator considered them unrelated to the study product.

Conclusions: ThermaCare Heatwraps provided significantly faster pain relief than oral placebo in subjects with muscular LBP. The double stopwatch is a viable approach for assessing the onset of analgesia in LBP.

\section{Classifications of Drug Related Problems Discovered During Brown Bag Medication Reviews for Older Adults}

Ashley M Tocco', Feng Chang', Megan E Kleinow', Jamie M Hwang', Candice L Garwood', Hanan S Khreizat', Mary Beth O'Connell'

${ }^{\prime}$ Eugene Applebaum College of Pharmacy and Health Sciences, Wayne State University, Detroit, MI

${ }^{2}$ School of Pharmacy, University of Waterloo, Waterloo, ON

${ }^{3}$ Henry Ford Health System, Detroit, MI

${ }^{4}$ Henry Ford Piersen Clinic, Grosse Pointe Farms, MI

Rationale: Classifications, severity and value of drug related problems (DRPs) have not been well documented for community based medication reviews.

Objectives: To quantify and classify DRPs discovered during community based brown bag medication reviews and determine recommendation acceptance.

Methods: Medication reviews were conducted in 9 senior centers. A medication calendar and DRP recommendations were provided post session. A follow-up survey was done at 3 months to capture recommendation implementation. After two training sessions, two pharmacists (10 coding pairs) classified each DRP using a modified Pharmaceutical Care Network Europe (PCNE) Classification Scheme for Drug-Related Problems (V6.2; www.pcne.org), Severity of Error in Medication Order (AJHP 99;56:2449), and Value of Service (AJHP 99;56:2449-50) scales. Disagreements were resolved by two additional investigators. Descriptive statistics were utilized for quantification and Kappa coefficients were calculated with SPSS v19 to determine inter-rater reliability.

Results: Participant averaged $75.9 \pm 8.5$ years old (range 60-93) with 4.3 \pm 2.8 DRPs per patient (range 0-10). Most common DRPs were related to adverse reactions $(30 \%)$, treatment effectiveness $(28 \%)$, other $(21 \%)$, treatment costs $(13 \%)$, and information (8\%). Top DRP causes were drug selection (40\%), dose (23\%), patient issues (16\%), drug use process 
(12\%), and other (7.4\%). Interventions required drug changes (44\%), prescriber input $(37 \%)$, patient counseling $(18 \%)$, or other $(1 \%)$. Seniors implemented $54 \%$ of the recommendations at follow-up. Most DRP severities were classified as significant $(59 \%)$ or minor (35\%). Most recommendation values were classified as significant $(50 \%)$ or somewhat significant (46\%). Kappa coefficients were PCNE problem (0.573), PCNE cause (0.39), PCNE intervention (0.728), severity (0.231), and value (0.176).

Conclusion: Senior medication reviews identified important DRPs with about half of the recommendations implemented at 3-months. Inter-rater reliability with the scales was quite variable, which might be corrected with more training and enhanced explanation of individual scale subdomains, especially for severity and value.

\section{Evaluation of Education on Preventive Care and Poison Control Center Awareness During Brown Bag Medication Reviews for Older Adults}

Nishi S Guptal, Feng Chang', Megan E Kleinow', Jamie M Hwang?,

Candice L Garwood', Hanan S Khreizat', Mary Beth O'Connell ${ }^{4}$

${ }^{1}$ School of Pharmacy, University of Waterloo, Waterloo, ON

${ }^{2}$ Henry Ford Health System, Detroit, MI

${ }^{3}$ Henry Ford Piersen Clinic, Grosse Pointe Farms, MI

${ }^{4}$ Eugene Applebaum College of Pharmacy and Health Sciences,

Wayne State University, Detroit, MI

Rationale: Preventive health is part of best practice for senior care but integration with medication review programs has not been examined.

Objectives: To evaluate education on preventive care and poison control center awareness achieved through brown bag medication reviews.

Methods: Individual medication reviews were conducted in 9 urban senior centres. Participants were educated on preventive care measures and provided with personalized recommendations. Participants were informed about utilizing poison control center for unintended medication ingestion and provided with magnets or stickers with the center's contact number. A follow-up survey was completed 3 months after. Unreturned surveys were followed up by telephone. Descriptive statistics were used.

Results: A total of 372 recommendations were made for 76 participants. Sixty-four (84\%) completed follow-up. At 3-months, the implementation rate was $38 \%$ with another $21 \%$ wanting to implement. Thirty-nine percent were willing to get the flu vaccine based on the pharmacist's recommendations; $75.6 \%$ considered getting or already got the pneumonia vaccine; $71.2 \%$ increased or wanted to increase their daily calcium intake; $67.3 \%$ were taking or considering taking vitamin $\mathrm{D}$; and $68.8 \%$ started taking a multivitamin or were considering to. An appreciable $75.4 \%$ of the seniors had already discussed or were considering discussing the pharmacist's recommendations with their physicians. Prior to the medication review, $17 \%$ of the seniors did not know how to act in case of accidental medication ingestion compared to $0 \%$ post session. Only $14.2 \%$ of the seniors knew to call a poison control center compared to $37.9 \%$ post session. The session was rated as being highly educational and the poison control center tools very helpful.

Conclusion: Community based medication reviews can be an effective setting for pharmacists to help seniors implement preventive care measures. By incorporating poison control center education into the review, seniors became more aware and more prepared to handle accidental medication ingestion.

\section{Self-perceived Knowledge Gained and Satisfaction from Brown Bag Medication Reviews for Older} Adults

Nishi S Guptal, Feng Chang', Megan E Kleinow', Jamie M Hwang', Candice L Garwood', Hanan S Khreizat', Mary Beth O'Connell ${ }^{4}$

${ }^{1}$ School of Pharmacy, University of Waterloo, Waterloo, ON

${ }^{2}$ Henry Ford Health System, Detroit, MI

${ }^{3}$ Henry Ford Piersen Clinic, Grosse Pointe Farms, MI

${ }^{4}$ Eugene Applebaum College of Pharmacy and Health Sciences,

Wayne State University, Detroit, MI

Rationale: Community based brown bag medication reviews are used in settings without access to primary care providers or the medical chart.

Objectives: To examine self-perceived knowledge gained and participant satisfaction during community based brown bag medication reviews.

Methods: Reviews were conducted in 9 senior centers for adults over the age of 60 and on 5 or more medications. Each appointment was scheduled for $45-60$ minutes. A medication calendar and a list of recommendations were given. Seniors were asked to complete a 13-items satisfaction questionnaire following the session and rate the program's helpfulness in a follow-up survey at 3 months. Each item was ranked 1-5 (strongly disagree - strongly agree). Self-perceived learning was reported using an 8-items survey. Each item was ranked 1-5 (very littlelots). Unreturned surveys were followed-up over the phone. Descriptive statistics were used.

Results: Sixty-four participants completed follow-up. All items on the satisfaction questionnaire were ranked between 4.44- 4.90. The highest ranked items were: having enough time to ask questions (4.90); time and place of the review were convenient (4.83); trust in the pharmacist's answers (4.83); and better understanding of their medications (4.83). The lowest ranked item was perceived resolution of their health problems (4.44). At 3-months, 98\% ranked the brown bag program as helpful. Self-perceived knowledge gained was rated between 4.40-4.71. The highest ranked items were: learning how to use their medications (4.71); when to correctly take the medications (4.66); and learning the purpose of their medications (4.64). Perceived knowledge gained in understanding medication side effects was rated the lowest (4.40). Over $95 \%$ would like to attend the program again.

Conclusion: Seniors were highly satisfied with the brown bag medication reviews. They felt a high degree of learning and found the program convenient. Individual items ranked demonstrated unique advantages and limitations of such programs.

\section{Cost Analysis of an Insulin Pen Pilot Project in an In-Patient Hospital Setting \\ Sara Kynicos', Jin Hyeun Huh ${ }^{l, 2}$ \\ ${ }^{1}$ University Health Network, Toronto, ON \\ ${ }^{2}$ Leslie Dan Faculty of Pharmacy, University of Toronto, $O N$}

Rationale: Following medication incidents involving insulin, a known high-risk medication, a 3 month pilot project using insulin pens for inpatients was implemented in general internal medicine ( 98 beds across 3 floors) at one hospital site.

Objective: To review costs and wastage associated with implementing insulin pens, together with nursing and safety benefits using staff surveys and a review of medication incidents.

Method: All existing formulary insulins were made available in pen devices as floor-stock and each patient was allocated their own pen for each insulin to be administered by nurses. A review of data compared the usage and costs of insulin dispensed during the 3-month pilot period to the 3 previous months of traditional $10 \mathrm{~mL}$ insulin vial dispensing. 
Results: The results showed that there was a $12 \%$ decrease in total volume ( $\mathrm{mLs}$ ) of insulin dispensed during the pen pilot, however less favourable contract prices resulted in increased total cost for pre-filled pens / cartridges, equivalent to a $24 \%$ increase ( $\$ 115$ per month per floor). During the pilot $20 \%$ more safety needles were used compared to traditional safety syringes. A review of insulin pen wastage over one month showed that $64 \%$ of insulin not used in the pen devices was short or rapid insulin types. No medication incidents involving insulin were identified during the pilot and nursing staff surveys indicated that they felt nursing time was saved due to pens being readily available for individual patient-use. Staff also suggested that less time was spent on discharge patient education, resulting in an improved patient discharge process.

Conclusion: This project demonstrates that insulin pen devices can be successfully implemented in an in-patient hospital setting, with medication safety, nursing time and patient discharge benefits, despite increased medication costs.

\section{Venous Thromboembolism Prophylaxis Electronic Physician Risk Assessment Tool \\ Pauline Santora, Cristina Scherf \\ Baycrest, Toronto, ON}

Rationale: VTE prophylaxis is the number 1 strategy to improve patient safety in hospitals and is recognized by Accreditation Canada as an 'organizational required practice'. Universally starting prophylaxis on admission did not seem justifiable at Baycrest, a geriatric acute, complex continuing care and long-term care facility where the situation is often more long term and risks of harm from the prophylaxis elevated.

Description of Concept: To develop an electronic tool that would provide a physician assessment of VTE prophylaxis for each geriatric hospitalized patient and that could electronically generate preselected quality indicators.

Steps Taken: Based on a literature review and manual chart audits, a multi-disciplinary group developed an algorithm with input from experts. Background information specific to geriatrics is available for each question at the click of a button. Information Technology built the algorithm into Meditech for easy access by the admitting physician who completes it within 24 hours of admission, transfer or new acute medical illness in the hospitalized patient. The policies, procedures, and algorithm were approved prior to training and implementation.

Evaluation: Physician's evaluation indicated that this electronic point and click tool is straight forward and can be completed in 15-60 seconds. The tool provides the electronic means to audit VTE prophylaxis assessment rates, the timeline of assessment completion, which VTE protection was ordered and if not, the reason(s), for any period, on any day, within minutes. Previously documented VTE Risk Assessments were hard to find. The electronic tool has increased this to $68 \%$ of admissions in May, $80 \%$ in June and $92 \%$ in July.

Importance to Practice: The use of technology to provide a practical standardized approach for VTE prophylaxis, supported by educational information and up-to-date audit results is extremely useful in our efforts to improve the safety of hospitalized elderly patients.

\section{Fingertip Sampling is a More Sensitive Evaluation of Aseptic Technique Competency}

Mari Culotta-Mascioli, John Iazzetta, Scott Walker, Fausto Noce

Sunnybrook Health Sciences Centre, Odette Cancer Centre, Department of Pharmacy, Toronto, $O N$

Rationale: A system of random gloved finger tip testing in tandem with a media fill test allows auditors to visually inspect aseptic technique in addition to producing evidence of positive or negative growth of microbes through finger tip imprinting on agar plates filled with test media.

Objectives: To determine microbial contamination rate during sterile preparation with the best of controls being in place. Compare these results with those from planned media fill tests done as part of an annual recertification.

Study Design: We conducted gloved finger tip sampling in 32 sterile compounders. Raised test plates containing trypticase soy agar with polysorbate and lecithin were used as the test media. Samples of gloved finger tips were taken under three scenarios. A group of 18 volunteers gave finger tip samples without having performed a scrub or hand hygiene. A second group of 16 after thorough hand scrubbing and a third group of 16 under random conditions while performing regular aseptic compounding duties. The samples collected were incubated for 48 hours at $33-37^{\circ} \mathrm{C}$. After 48 hours the plates were visually inspected for colony forming units. The Media fill testing was conducted during annual recertification.

Results: After the incubation period it was noted that while some plates resulted in no growth, $62 \%$ of plates from all three groups were positive with CFU's. However, all the volunteers were able to produce test media bags with no growth at time of planned testing in previous tests.

Conclusion: Our results indicate a need for an additional evaluation to ensure a high standard of aseptic technique is maintained. Perhaps an evaluating system that allows for more frequent random auditing

\section{Potential Cross-reactivity between Prasugrel and Clopidogrel}

Joyce Chan ${ }^{I}$, Yvonne Kwann ${ }^{I}$, Sonia Mota ${ }^{I}$, Kori Leblanc ${ }^{l, 2}$

${ }^{1}$ University Health Network, Toronto, $\mathrm{ON}$

${ }^{2}$ Faculty of Pharmacy, University of Toronto, Toronto, ON

Rationale: Prasugrel is a third-generation thienopyridine antiplatelet agent effective for the management of acute coronary syndrome in planned percutaneous coronary intervention (PCI). It became available to Canadian patients in June 2010 and is attractive as an alternative in patients who have experienced adverse reactions to clopidogrel. Crossreactivity between clopidogrel and other thienopyridine antiplatelet agents has been reported. Although cross-reactivity with prasugrel on allergy testing has been seen, reports of patient experience are extremely limited.

Objective: To document the experience of patients with a history of adverse skin reactions to clopidogrel who received prasugrel.

Methods: Patients with a documented skin reaction to clopidogrel who were prescribed prasugrel were followed at $24 \mathrm{~h}$ and post-discharge. Data was collected regarding tolerance as well as adherence to therapy.

Results: Eight patients received prasugrel. All reported good medication adherence. One patient developed an itchy macular rash over the body and upper thighs within 24 hours of prasugrel exposure, requiring discontinuation and replacement with ticlopidine. A Naranjo Adverse Drug Reaction (ADR) probability score of 4 defined this as a "possible" ADR association. No other patients experienced skin reactions. Seven out of 8 patients required financial assistance to afford prasugrel which was facilitated by the pharmacist pre-discharge. In all cases, the patients' community pharmacy did not have prasugrel readily available. 


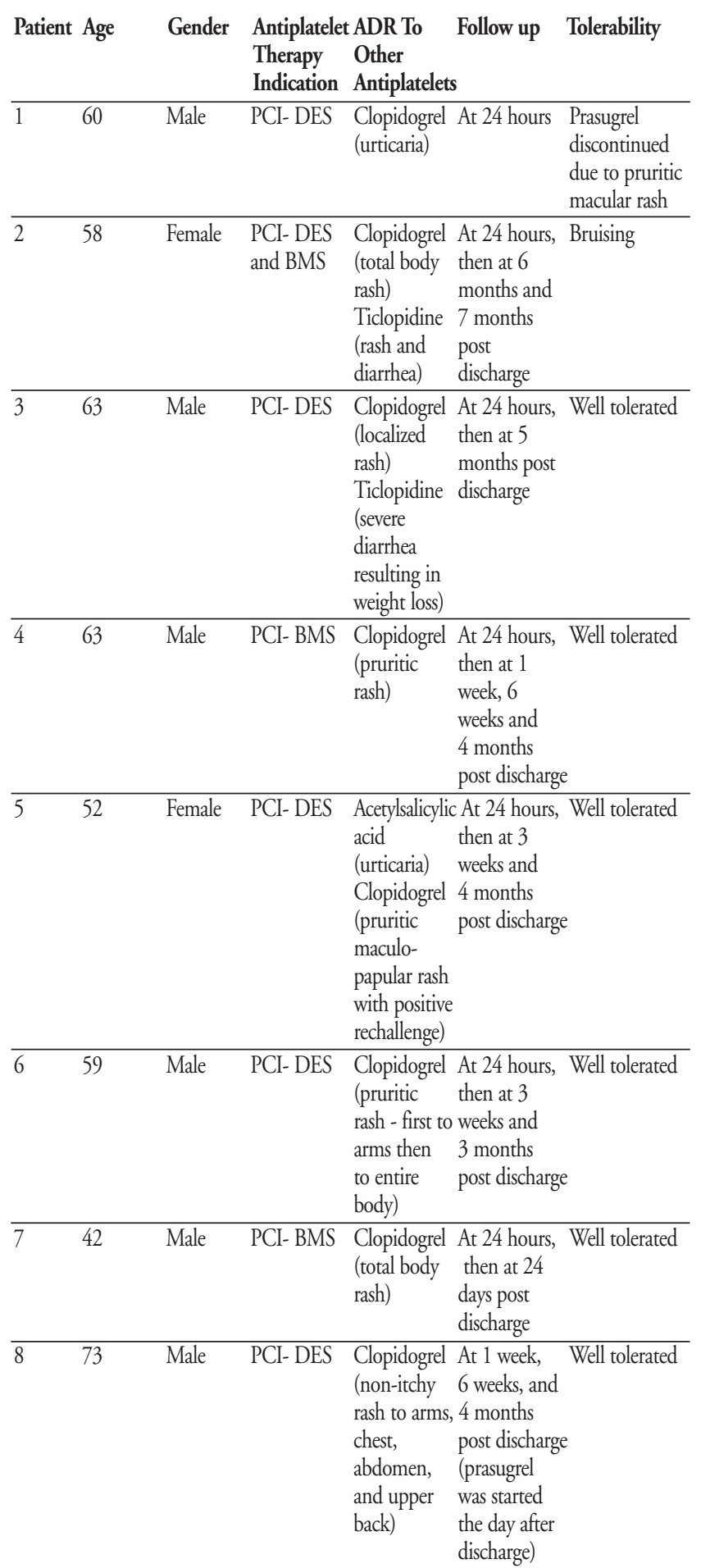

DES: Drug Eluting Stent

BMS: Bare Metal Stent

Conclusion: Our experience with 8 patients with previous skin reactions to clopidogrel suggests that there is at least a small risk of cross-reactivity with prasugrel. Further experience is needed to clearly define that risk. Determining patients' financial coverage for prasugrel and facilitating its availability in community pharmacies post-discharge is essential to ensure that doses are not unintentionally missed.

\section{Total Parenteral Nutrition: Supporting Practice Through Standardized Solutions}

Rosemary Tanzini, Jill Garland

St Michael's Hospital, Toronto, ON

Rationale: Commercial options for TPN base solutions no longer met evolving practice. Over the years, in order to meet protein requirements, the administration of TPN had become more complex, due to the additional administration of amino acids (Y-connected in a separate bag to the 2-in-1 (dextrose/amino acid) bsolutions. This "modular" approach to supplying TPN complicates the prescribing process and increases the risk of pump programming errors and workload for the RN. Lack of standardization and a manual system of generating patient specific worksheets and labels for compounding purposes was also labor intensive for Pharmacy and increased the risk of errors. It also became apparent that to enable electronic compounding worksheets automated for calculations, it was necessary to provide the daily dextrose/amino acid requirement into 1 bag. Developing a "menu" of TPN base solutions which would meet the needs of the majority of patients and a comprehensive TPN order form has the potential to facilitate prescribing, production and administration.

Description/Implementation: Project began with audit of current state to determine different permutations of amino acids/dextrose/lipids being prescribed, as well as wastage. Empiric calculations, for the purpose of dose banding, was done for patients ranging in weight, taking into account target calories and step-wise approach to advancing caloric intake in patients at risk of refeeding syndrome. Next, there was a review of commercial and compounding options (with stability data), including a time-motion study. Input from Nutrition team was critical to coming up with a final list of solutions. Accordingly, an order set was developed which clearly outlined options and provide decision support. Electronic compounding worksheets and computer-generated labels were to follow. Evaluation: Over first 5 months post implementation, of 53 patients, standard solutions met the needs of all but 2 patients.

Importance to Practice: Improved safety, quality, transparency, efficiency and communication.

\section{Determination of Tobramycin Pharmacokinetics in Burn Patients to Evaluate the Potential Utility of Once Daily Dosing in this Population}

Diane Vella, Sandra A N Walker, Scott E Walker, Andrew Simor Sunnybrook Health Sciences Centre, Toronto, ON

Rationale: Once-daily aminoglycosides (ODAs) have been avoided in burn injury patients, due to their complicated pharmacokinetics. Theoretically, ODAs may have the same advantages in burn patients as in other populations.

Objectives: The objectives were to determine the pharmacokinetics of tobramycin in adult critically ill burn patients and evaluate a variety of $\mathrm{mg} / \mathrm{kg}$ dosing regimens to determine if ODA therapy was feasible.

Methods: A retrospective study of 58 adult critically ill burn injury patients who received tobramycin and had at least one set of tobramycin steady-state levels was conducted. Pharmacokinetic parameters were calculated using first-order pharmacokinetic equations. CART analysis to identify whether a breakpoint for days post burn injury existed for tobramycin clearance $(\mathrm{Cl})$ and Monte Carlo Simulations (MCS) of a range of $\mathrm{mg} / \mathrm{kg}$ dosing regimens, using mean pharmacokinetic parameters with respective standard deviations targeting a peak of $\geq 20 \mathrm{mg} / \mathrm{L}$ and trough $<2 \mathrm{mg} / \mathrm{L}$ with at least $70 \%$ probability were run.

Results: A CART identified breakpoint of days post burn for $\mathrm{Cl}$ of $\leq 45$ days versus $>45$ days was determined. The geometric mean $(95 \% \mathrm{CI})$ pharmacokinetic parameters in patients receiving tobramycin: $\leq 45$ days post-burn were: $k e 0.167 \mathrm{~h}^{-1}(0.15-0.18), t_{1 / 2} 4.1 \mathrm{~h}(3.8-4.6), V d 0.40 \mathrm{~L} / \mathrm{kg}$ 
(0.37-0.43), $\mathrm{Cl} 5.3 \mathrm{~L} / \mathrm{h}$ (4.9-5.8); and >45 days post-burn were: ke $0.13 \mathrm{~h}^{-1}$ (0.11-0.14), t $t_{1 / 2} 5.4 \mathrm{~h}$ (4.8-6.1), Vd 0.37L/kg (0.32-0.41), Cl 3.7L/h (3.2-4.1). MCS identified ODA regimens that attained the desired targets using higher $\mathrm{mg} / \mathrm{kg}$ dosing known to be safe in other populations ( $\leq 45$ days post-burn: 10 to $13 \mathrm{mg} / \mathrm{kg}$; $>45$ days post burn: 8 to $10 \mathrm{mg} / \mathrm{kg}$ ).

Conclusion: This study identified initial ODA tobramycin dosing recommendations that could attain desired targets in burn patients.

\section{Diagnostic Reasoning by Hospital Pharmacists: An Assessment of Attitudes, Knowledge, Skills, and Learning Needs \\ Kseniya Chernushkin, Peter Loewen, Joanne Jung, Amneet Aulakh, \\ Jane de Lemos, Karen Dahri \\ Vancouver General Hospital, Vancouver, BC}

Background: Currently, hospital pharmacists participate in activities that can be seen as diagnostic in nature. Two reasoning approaches to making diagnoses have been previously described: non-analytic and analytic. Of the six analytic traditions "probabilistic" has been shown to improve diagnostic accuracy and reduce unnecessary testing. Pharmacists' attitudes toward having a diagnostic role and their diagnostic knowledge and skills have never been studied.

Objectives: To describe pharmacists' attitudes toward the role of diagnosis in pharmacotherapeutic problem-solving; to characterize the extent of pharmacists' knowledge and skills related to diagnostic literacy; to identify knowledge/skill gaps and propose means of closing them.

Method: Pharmacists of Lower Mainland Pharmacy Services with at least $33 \%$ in direct patient care were studies in a prospective observational survey.

Results: A sample of 260 pharmacists was identified. Results showed that $89 \%$ of participants agreed with the proposed definition of diagnosis and $92 \%$ agreed that it is important for pharmacists to have skills related to diagnosis. Respondents preferred analytic to non-analytic approach to diagnostic decision-making. Probabilistic tradition was not the preferred method in any of the three cases. In the evaluation of clinical scenarios that may require diagnostic skills $84 \%$ of respondents agreed that they should be involved in assessing such problems and $84 \%$ agreed that the case problems were diagnostic in nature. Participants' knowledge of and ability to apply probabilistic diagnostic tools were highest with test sensitivity (61\% of correct answers) and lower with test specificity (48\% of correct answers) and likelihood ratios (39\% of correct answers).

Conclusions: The pharmacists studied strongly believe that diagnostic skills are important for them to solve drug-related problems, but demonstrated low levels of knowledge and ability to apply concepts of probabilistic diagnostic reasoning. Opportunities to expand pharmacists' knowledge of diagnostic reasoning exist, and our findings indicate that they would consider such development valuable.

\section{A Pilot Study on the Use of Warfarin Sliding Scales in Hemodialysis Patients \\ Grace Leung, Emmelia Lau, Henry Chen \\ York Region Chronic Kidney Disease Program, York Central Hospital, Richmond Hill, ON}

Background: Inter-prescriber variation is one of the causes of INR fluctuations in patients on warfarin. The use of a pharmacist-driven warfarin sliding scale (WSS) has the potential to decrease inter-prescriber variation to achieve more stable INRs within the therapeutic range. This pilot study investigates the use of WSS in hemodialysis patients at a multi-site dialysis program with weekly nephrologist rotations.
Methods: In this self-controlled study, 27 hemodialysis patients stable on warfarin with a target INR of 2-3 were selected to have warfarin dosed by WSS. Pharmacists designed and adjusted the WSS based on previous INR results and warfarin doses with nephrologist approval. INR results 6 weeks before and 6 weeks after the implementation of WSS were compared. The primary outcome was percentage of time with a therapeutic, sub- (INR < 1.5) and supra-therapeutic (INR > 4) INR. Secondary outcomes include bleeding or clotting events. Statistical analysis was performed with Wilcoxon Paired Signed Rank Test. A p-value of $<0.05$ was considered statistically significant.

Results:

\begin{tabular}{llll} 
& $\begin{array}{l}\text { \% INR, } \\
\text { traditional dosing }\end{array}$ & \% INR, WSS & P-value \\
& 50.7 & 59.7 & 0.0018 \\
\hline INR 2-3 & 9.7 & 8.1 & 0.40 \\
\hline INR $<1.5$ & 4.2 & 1.2 & 0.001 \\
\hline INR $>4$ & & &
\end{tabular}

The use of WSS resulted in significantly more therapeutic INRs versus traditional dosing. Supra-therapeutic INRs were also reduced significantly and sub-therapeutic INRs were trending lower.

Discussion: Pharmacists provided consistency in warfarin dosing via WSS by avoiding drastic changes in warfarin doses as a result of overreaction to sub- or supra-therapeutic INRs. Nursing and physician time was saved as nurses no longer need to call physicians about patients' INRs. Conclusions: More therapeutic INRs are achieved by pharmacists' involvement in warfarin dosing via the WSS, which has positive impact for patients, nurses and nephrologists. WSS use can be considered for other hemodialysis patients stable on warfarin.

\section{Potentially Inappropriate Medication Use In Elderly Patients Presenting to the Emergency Department \\ Vincent Teo ${ }^{I}$, Patrick Edwards ${ }^{2}$, Minh-Hien Le $e^{I}$ \\ ${ }^{1}$ Sunnybrook Health Sciences Centre, Toronto, ON \\ ${ }^{2}$ Leslie Dan Faculty of Pharmacy, University of Toronto, $O N$}

Rationale: The Beers criteria is a list of medications considered inappropriate in elderly patients and it has been widely used to assess the safety of medications in ambulatory and nursing facilities. Currently, few studies have examined the prevalence of potential inappropriate medication use in elderly patients presenting to the emergency department (ED).

Objective: To determine the incidence of potentially inappropriate medication use in elderly patients presenting to the ED at Sunnybrook Health Sciences Centre.

Methods: Patients 65 years of age or older who were eligible for coverage by Ontario Drug Benefit (ODB) were included in the study. Patients were excluded if they had removed consent from the ODB drug profile viewer (DPV). A current medication list for each patient was obtained from the DPV and demographic information for each patient was collected from the ED chart. Using the Beers criteria, medication lists were assessed for potentially inappropriate medications independent of a diagnosis or condition.

Results: Data were collected for 60 consecutive days resulting in a total of 2940 patients who met the inclusion criteria. A total of 252 patients (8.6\% of eligible ED visits) were found to be taking at least one potentially inappropriate medication when they presented to the ED. Nineteen patients were taking two potentially inappropriate medications and one patient was taking three. The three most frequently occurring potentially inappropriate medications were amiodarone ( 50 instances), followed by amitriptyline and nitrofurantoin (44 and 39 instances, respectively).

Conclusion: Although this study did not determine whether the potentially inappropriate medications were the cause for a patient's presentation to the ED, it highlights the need for increased awareness concerning safe medication prescribing practices in the elderly. 


\section{Intentional Overdose of Enoxaparin}

Koren Lui $i^{1,2}$, Vincent Teo ${ }^{3}$

${ }^{1}$ Canadian Forces Health Services Group

${ }^{2}$ Leslie Dan Faculty of Pharmacy, University of Toronto, Toronto, ON

${ }^{3}$ Sunnybrook Health Sciences Centre, Toronto, ON

Rationale: Minimum toxic doses of low molecular weight heparins (LMWH) are not well established. Published cases of LMWH overdose have reported consumption of up to 30-times the therapeutic dose. Overdoses of LMWH are rarely fatal and there are no clear guidelines pertaining to the management of acute LMWH overdoses. A case of acute intentional enoxaparin overdose is reported.

Description of Case: A 42 year-old male presented to the emergency room claiming he had injected himself with a 10-day supply of enoxaparin. He exhibited signs of intoxication and also complained of leg pain. The patient's medical history included bilateral deep vein thrombosis, pulmonary embolism, hepatitis B, cholecystitis, ethanol abuse, and suspected tetrahydrocannabinol (THC) use. His medications prior to admission consisted of enoxaparin $100 \mathrm{mg}$ subcutaneous injection daily. On examination, it was noted that the patient lacked motor coordination. There were no signs of bleeding. His INR (1.23) and aPTT (97.1 s) were elevated. Poison control was contacted and suggested hourly monitoring of aPTT, INR, and vital signs until the patient's INR and aPTT normalized.

Assessment of Causality: Temporal relationship suggests that the elevated INR and aPTT were the result of acute LMWH overdose.

Evaluation of Literature: At time of writing, three published case reports of acute intentional LMWH overdose were found. In all cases, the patients did not experience bleeding. The aPTT's were monitored until normalized. A published analysis of available case reports suggested that management by observation is appropriate.

Importance of Case to Pharmacy Practitioners: There are available antidotes for the anticoagulant activity of LMWH. However, a more appropriate course of action may be to observe and monitor for signs of bleeding until the aPTT is normalized. It is not necessary to treat with protamine or factor VIIa unless there is an active bleed in the setting of LMWH overdose.

\section{Implementation of a Pharmacy Residency Program in Primary Care \\ Debbie Kwan ${ }^{1,2}$, Karen Cameron ${ }^{l, 2}$, Patricia Marr ${ }^{1,2}$, Christine Papoushek ${ }^{1,2}$, Victoria Siu ${ }^{1,2}$ \\ ${ }^{1}$ University Health Network, Department of Pharmacy, Toronto, ON \\ ${ }^{2}$ Leslie Dan Faculty of Pharmacy, University of Toronto, ON}

Rationale: Integration of pharmacists into primary care has been identified as a priority for primary health care reform in Canada. However, little exists by way of formal training to prepare pharmacists for this role.

Description: The Toronto Western Hospital Academic Family Health Team (FHT) serves approximately 14,000 patients in downtown west Toronto. In 2008, the FHT launched Canada's first Pharmacy Residency Program in Primary Care. The goal of the program is to provide practicebased, experiential learning to prepare residents to develop expertise and successful careers as pharmacists in primary care. The resident works with patients and in collaboration with members of the inter-professional team. Required and ambulatory-based elective rotations are completed longitudinally. The resident is also required to complete a primary care focused research project, teach health professional students and give academic presentations.

Implementation: The application process for this residency program was linked to the same application process for hospital pharmacy residency programs in Ontario and was open to graduates of post-baccalaureate pharmacy programs. As such, the application and interview process commenced in the fall of 2007 and the first resident was accepted into the program starting July 2008.

Evaluation: Two candidates have successfully completed the program. One graduate continues to work in the Toronto Western FHT. The number of applications to the program has risen yearly indicating an ongoing demand for such a program.

Importance: As the pharmacist's role in primary care continues to grow, training for practitioners in this unique practice area will continue to be required. In addition, the implementation of new residency programs helps to fill the increasing demand for pharmacy residency training in Canada.

\section{Differences in Nova Scotian Pharmacists' Experiences and Barriers to Providing the Emergency Contraceptive Pill Based on Primary Practice Site \\ Anne Marie Whelan', Donald Langille ${ }^{2}$, Eileen Hurst, ${ }^{1}$ \\ ${ }^{1}$ College of Pharmacy Dalhousie University, Halifax, NS \\ 'Department of Community Health \& Epidemiology, Dalhousie University, Halifax, NS}

Rationale: There is little in the literature about differences between pharmacists whose primary place of practice is hospital versus community, pertaining to their experiences and barriers to providing the emergency contraceptive pill (ECP).

Objectives: In 2005 ECP became more accessible to women in Canada when the regulatory status of levonorgestrel changed from Schedule I to Schedule II (available without a prescription in pharmacies following a consultation with a pharmacist). The objective of this study was to examine if there is a difference between primarily hospital based versus community based pharmacists' experiences providing ECPs.

Study Design and Methods: All pharmacists licensed to practice in Nova Scotia were invited to anonymously complete a 25 item paper questionnaire sent in the mail. The research was approved by Dalhousie University Health Sciences Ethics Board. Descriptive statistics were generated using SPSS.

Results: The response rate was 53\% (595/1123) with 415 pharmacists indicating that they had previously provided nonprescription ECP in community pharmacy and their primary place of practice was either hospital or community (13 hospital, 402 community). In comparing their experiences providing ECP, $38.7 \%$ of hospital pharmacists indicated they spent 11 minutes or longer providing consults while only $17.4 \%$ of community pharmacists spent this much time. With regard to barriers to ECP provision, more hospital pharmacists than community pharmacists reported the following as barriers: 1) lack of privacy $(61.6 \%$ vs. $46.0 \%), 2)$ lack of staffing $(76.9 \%$ vs. $49.6 \%)$, and 3$)$ length of consultation ( $46.2 \%$ vs. $28.0 \%$ ).

Conclusion: Results from this survey suggest that there may be some differences in the experiences between hospital and community based pharmacists providing ECP which should be explored further. Regardless of primary practice site, results indicate that there are some barriers to ECP provision that should be addressed when considering providing this type of consultation in a community pharmacy. 


\section{Chemotherapy-Induced Nausea and Vomiting in Children Receiving Ifosfamide plus Etoposide: Preliminary Results \\ AS R Lavoratore, C Lui, M Linseman, A M Maloney, P C Nathan, TTaylor, E Zelunka, L L Dupuis \\ The Hospital for Sick Children, Toronto, $O N$ \\ Leslie Dan Faculty of Pharmacy, University of Toronto, Toronto, ON}

Rationale: Adult evidence is commonly used to determine the emetogenicity of chemotherapeutic agents in children and informs the selection of nausea and vomiting prophylaxis. This approach may be inaccurate in children. Pediatric information regarding the emetogenic potential of ifosfamide plus etoposide, a combination which in adults is deemed to be moderately emetogenic, is lacking.

Objective: To describe chemotherapy-induced nausea and vomiting (CINV) in the acute and delayed phases in children receiving ifosfamide $1800 \mathrm{mg} / \mathrm{m}^{2} /$ day plus etoposide $100 \mathrm{mg} / \mathrm{m}^{2} /$ day for 5 days.

Study Design and Methods: A diary was used by children to record episodes of vomiting and retching, nausea severity and antiemetic agents administered during acute and delayed phases. Patients themselves reported nausea severity a minimum of twice daily using a validated pediatric nausea assessment instrument. During the phase of interest, complete CINV control was defined as no emetic episodes (no vomiting or retching) and a maximum nausea score of 1 (none) out of 4 . Patients received antiemetic agents as prescribed by the clinical team.

Results: 10 patients (median age: 10.1yrs; range: 5-17yrs) participated. No patient was chemotherapy-naïve. Three had a history of motion sickness and 2 experienced anticipatory CINV. All patients received ondansetron during the acute phase; 9 also received dexamethasone. Four patients experienced complete acute phase CINV control. Six patients received ondansetron during the delayed phase while 2 also received dexamethasone. Six patients experienced complete CINV control during the delayed phase.

Conclusions: Preliminary findings suggest that ifosfamide plus etoposide is highly emetogenic in children. Interventions aimed at improving CINV control in both the acute and delayed phases should be investigated. Study findings are limited by small sample size.

\section{Improving a Complex Pharmacist Scheduling Model at a Tertiary Care Hospital}

Daniel Cortes, Kevin Curley, Jodie Leung, Jenny Lieu St. Michael's Hospital, Toronto, $O N$

Rationale: Pharmacist scheduling has undergone increased strain and demand amidst recent medication system changes such as a computerized physician order entry system and expansion to 24-hour pharmacy operations. In this new environment, the complexities of scheduling for 25 pharmacists necessitated the development of a new scheduling model that would decrease workload for schedulers, while meeting the needs of individual pharmacists.

Description: A literature review on MEDLINE and CINHAL provided guidance based on nursing scheduling models. A hybrid model of selfscheduling and set-scheduling was identified, that allows for autonomy, flexibility, fairness and transparency for pharmacists and decreased workload for schedulers. An Excel-based scheduling tool was developed that allows pharmacists' availability based on cross-coverage responsibilities, vacations, teaching and professional development responsibilities to be entered. This data can then be used to ensure all assigned shifts are covered, shifts are equally distributed and that each pharmacist meets their quota of required shifts.

Evaluation: Post-implementation, pharmacists were surveyed to assess if the new model met their needs based on customizability, flexibility, fairness, transparency and effort required. A total of 23 pharmacists responded and the ratings for the new model met or exceeded ratings for the prior model in all categories. Overall, 21 of the pharmacists ( $92 \%$ of respondents) reported being somewhat or very satisfied with the new model. In contrast, $70 \%$ of eligible respondents were dissatisfied with the previous scheduling model. Schedulers found a reduced administrative burden for filling vacant shifts and decreased time needed to create the schedule.

Importance: The new scheduling model provides decreased workload for schedulers while meeting individual clinical pharmacists' needs.

\section{Statin Treatment Use in Diabetics with Breast Cancer: A Potential C-Reactive Protein Mediated Benefit \\ A C Ceacareanu, C C Hong, J J Brennan III, M Epstein, E Kossoff, $G K$ Nimako, K Patel, A Forrest \\ School of Pharmacy and Pharmaceutical Sciences, State University of New York, Buffalo, $N Y$ \\ NYS Center of Excellence, Buffalo, NY \\ Roswell Park Cancer Institute, Buffalo, NY}

Background: PStatin treatment has not yet been evaluated in relation to breast cancer (BC) prognosis in diabetes mellitus (DM) patients. Reported decreased survival following $\mathrm{BC}$ in women with metabolic syndrome, led us to investigate whether specific statin therapy may lead to improved BC survival. Reported associations between elevated C-reactive protein (CRP) levels at the time of diagnosis with worse cancer prognosis, and CRP-lowering properties of statin treatment in non-cancer patients, led us to investigate whether statin use is associated with lower CRP levels at the time of $\mathrm{BC}$ diagnosis and with improved $\mathrm{BC}$ outcomes.

Methods: All DM patients newly diagnosed with BC between 2003 and 2007 (Roswell Park Cancer Institute) were retrospectively reviewed ( $\mathrm{n}=$ 225). BC pathology, outcomes, existing comorbidities and drug therapy were documented. Follow up began at $\mathrm{BC}$ diagnosis and ended with first confirmed recurrence and/or death, or last date of follow-up. Hazard ratios $(\mathrm{HR})$ and $95 \%$ confidence intervals $(\mathrm{CI}) \mathrm{s}$ representing the association between statins, $\mathrm{BC}$ and a defined event were computed with Cox proportional hazards model. CRP plasma levels were determined by enzyme-linked immunosorbent assay in specimens donated at the time of $\mathrm{BC}$ diagnosis. A total of 98 study patients, $\mathrm{DM}+\mathrm{BC}$, and their matched controls, BC only $(n=196)$ were analyzed.

Results: After a median follow up of 30 months, patients receiving statins for cholesterol management were found to have better disease-free survival (HR 0.27, 95\% CI: 0.10, 0.71, $\mathrm{X}^{2}=7.31, \mathrm{p}=0.06$ ), and lower overall mortality (HR 0.23, 95\% CI: $0.08,0.66, \mathrm{X}^{2}=7.80, \mathrm{p}=0.05$ ) compared to patients not receiving any cholesterol management medication. CRP levels have ranged between 0.2 and $21 \mathrm{mg} / \mathrm{L}$ and clinically relevant levels $(>3 \mathrm{mg} / \mathrm{L}$ ) were noted in the study group.

Conclusions: This study explored for the first time the association between statin therapy and $\mathrm{BC}$ prognosis in DM. We observed improved outcomes in statin-treated patients. While we currently analyze statin therapy in relationship with baseline CRP levels and BC prognosis, our existing findings suggest that statins have the potential to improve $\mathrm{BC}$ outcomes potentially through lowering overall inflammation. 